\title{
36. OLIGOCENE TO EARLY MIOCENE SILICOFLAGELLATES FROM THE IVORIAN BASIN, EASTERN EQUATORIAL ATLANTIC, SITE 9591
}

\author{
Maija E. Schellpeper ${ }^{2}$ and David K. Watkins ${ }^{2}$
}

\begin{abstract}
Silicoflagellate biostratigraphy and relative abundance were determined for Site 959 in the eastern equatorial Atlantic. Five silicoflagellate zones, Corbisema apiculata, Naviculopsis biapiculata, Naviculopsis lata, Naviculopsis ponticula, and Corbisema triacantha Zones, were used with the Distephanus speculum hemisphaericus Subzone occurring in the $N$. biapiculata Zone. The Naviculopsis quadrata Zone is missing between the $N$. ponticula and $N$. lata Zones, indicating a hiatus in the lower Miocene cores that corresponds to hiatus NH1. Silicoflagellate relative abundance was graphed as a measure of paleoproductivity for Hole 959A. One large pulse of productivity occurred in the latest early Miocene, right before silica deposition came to an abrupt halt in the earliest middle Miocene.
\end{abstract}

\section{INTRODUCTION}

Leg 159 has been one of the few legs of the Ocean Drilling Program to drill the eastern equatorial Atlantic. The objectives of the cruise were to study the evolutionary stages of continental transform margins and investigate deep and intermediate water influences in the eastern equatorial Atlantic throughout the development of the basin. From the Oligocene to the present, Site 959 has been at or near the equator, and therefore, the cores recovered offer an excellent opportunity to study the paleoceanographic history of the eastern equatorial Atlantic and to determine the characteristics of the water masses that were present. In this study, silicoflagellate relative abundance data were collected to define the biostratigraphy for the upper Oligocene to the lower Miocene and investigate the paleoproductivity of the site.

Site 959 is located at $3^{\circ} 37.7^{\prime} \mathrm{N}, 2^{\circ} 44.1^{\prime} \mathrm{W}$ on the Ivory CoastGhana Marginal Ridge south of the Ivorian Basin (Fig. 1). Cores from Site 959 provide a sediment record from the mid-Cretaceous to the present, with silicoflagellates occurring in the Oligocene to lower Miocene. Silicoflagellate preservation and abundance are excellent in the upper Oligocene to uppermost lower Miocene sediment, but downcore in the lower Oligocene, silicoflagellate preservation diminishes. Hole 959A consists of calcareous ooze in the upper $205 \mathrm{~m}$ (Sections 159-959A-1H-CC through 22X-CC) underlain by $237 \mathrm{~m}$ (Sample 159-959A-23X-1, 39-41 cm, to Sample 159-959A-46XCC) of interbedded nannofossil ooze and biosiliceous ooze. Twelve samples from the upper portion of Hole 959D (Samples 159-959D1R-CC through 7R-CC) contain poorly preserved, biogenic siliceous components with sparse nannofossils. Core recovery for this interval of Hole 959D was 16\%, which made age designations and identification difficult. Samples downcore from 159-959D-7R-CC consist of diagenetically altered porcellanite and chert and were not examined for silicoflagellates.

\section{METHODS AND SAMPLE PREPARATION}

Samples were prepared as smear slides on $22 \mathrm{~mm} \times 40 \mathrm{~mm}$ cover slips. The number of silicoflagellates was tabulated in 10 transects

${ }^{1}$ Mascle, J., Lohmann, G.P., and Moullade, M. (Eds.), 1998. Proc. ODP, Sci. Results, 159: College Station, TX (Ocean Drilling Program)

${ }^{2}$ Geology Department, University of Nebraska, 214 Bessey Hall, Lincoln, NE 68588-0340, U.S.A.00091355@bigred.unl.edu across the slide, which is equal to an area of $4.92 \mathrm{~cm}^{2}$, more than half of the slide area (Tables 1-4). The specimens were counted at 200x magnification, and a magnification of $400 \times$ was used for more precise identification. Only specimens with over half of the skeleton were counted. On a few slides, over 300 specimens were counted, but for most of the slides, 50-100 specimens were found in the 10 transects. Because aberrant specimens may be an indication of environmental stress, they were counted on a separate tally.

Silicoflagellates from Holes 959A and 959D were examined for biostratigraphic purposes. The slides from Hole 959A, ranging from lower Miocene to upper Oligocene, exhibit good silicoflagellate skeletal preservation. In sediments of the lower Oligocene, incomplete diatom and silicoflagellate skeletons show evidence of dissolution and diagenesis.

\section{BIOSTRATIGRAPHY}

The biostratigraphy used here is derived from the previously published zonations of Bukry (1981), Perch-Nielsen (1985), and Locker and Martini (1986). Silicoflagellate counts in the oldest cores of Hole 959A and all of Hole 959D are so low that it is difficult to obtain a good biostratigraphic record for the ?lower Oligocene to lowermost upper Oligocene.

\section{Corbisema triacantha Zone}

Definition: This zone is bracketed by the last occurrence of Naviculopsis to the last occurrence of Corbisema triacantha triacantha.

Author: Martini, 1971.

Common species: The common species in this zone include Dictyocha pulchella, Dictyocha fibula ausonia, Corbisema triacantha triacantha, Bachmannocena elliptica, and D. fibula ausonia (naviculopsoid). Distephanus speculum hemisphaericus also is present in the lower part of the zone, but is not as common as the other listed species.

Remarks: The upper part of the $C$. triacantha Zone is not recognized in the sediments of Site 959, because there was a halt in siliceous preservation by the middle Miocene, before the last occurrence of $C$. triacantha. The last occurrence of Naviculopsis lies in Sample 159-959A-25X-CC. The minimum thickness of the zone is $27.2 \mathrm{~m}$.

\section{Naviculopsis ponticula Zone}

Definition: This zone is the interval defined by the first occurrence of Naviculopsis ponticula to the last occurrence of Naviculopsis.

Author: Bukry, 1981.

Common species: The common species include $C$. triacantha, $N$. ponticula, Distephanus crux, and B. elliptica. Other species include Distephanus speculum patulus, Distephanus speculum hemisphaericus, and Distephanus speculum pentagonus.

Remarks: This zone was found in Hole 959A with the first occurrence of N. ponticula in Sample 159-959A-29X-3, 38-40 cm, and the last occurrence 
Figure 1. Diagram showing the drilling sites (solid circles) of Leg 159. Site 959 is located on the CIG Marginal Ridge (Mascle, Lohmann, Clift, et al., 1996). Triangle is the location of Hole IVCO 2.

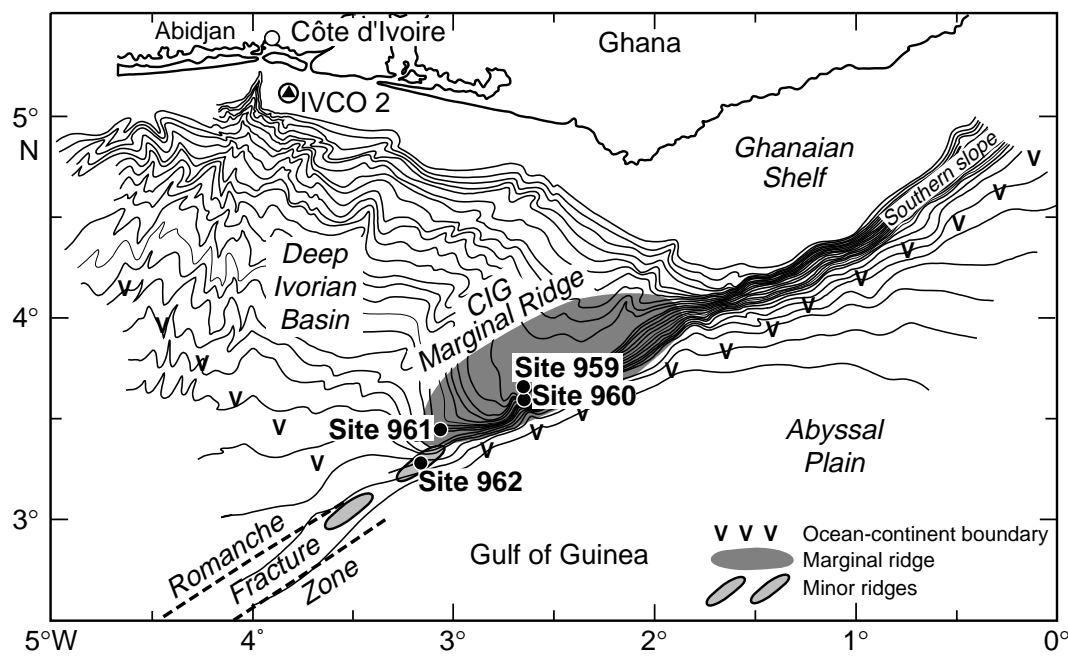

nus stradneri var. grandis, Ds. hannai, Ds. longispinus, Naviculopsis var. 2, Naviculopsis lata var. 1 , and $N$. constricta.

Remarks: Bukry (1981) originally described this interval as ranging from the first occurrence of Distephanus speculum haliomma and the first occurrence of Ds. speculum hemisphaericus to the first occurrence of $N$. lata. However, in this work only the first occurrence of Ds. speculum hemisphaericus was used as the lower boundary because Ds. speculum haliomma was not distinguished from Ds. speculum hemisphaericus. The first occurrences of Ds. speculum hemisphaericus and Ds. speculum speculum are in Sample 159959A-39X-5, 123-124 cm. The first occurrence of N. lata is in Sample 159959A-31X-5, 86-88 cm. Preservation was good throughout the subzone, which spanned a thickness of $75.8 \mathrm{~m}$.

\section{Corbisema apiculata Zone}

Definition: This zone is defined by the last occurrence of Dictyocha hexacantha to the last occurrence of Corbisema hastata hastata.

Author: Perch-Nielsen, 1975; emended, Bukry, 1978a.

Common species: In this zone the common species were C. hastata globulata, C. triacantha triacantha, $N$. biapiculata, $N$. constricta, B. apiculata apiculata, D. deflandrei, Naviculopsis biapiculata var. 2, and Ds. crux.

Remarks: The boundaries of this zone were difficult to accurately determine because silicoflagellates were sparse. Corbisema hastata hastata was not identified in any of the samples, so it can not be used as the zonal marker. Corbisema hastata globulata was identified in the cores and was used as the zonal marker in place of $C$. hastata hastata. The last occurrence of C. hastata globulata marks the top of the C. apiculata Zone in Sample 159-959A-42XCC. Because lower sediments in this zone consisted of porcellanite and chert, the bottom zonal marker of this zone was not determined. The minimum thickness of this zone is $75.8 \mathrm{~m}$.

\section{DISCUSSION}

The missing $N$. quadrata Zone marks a disconformity in the lower Miocene sequence. As discussed earlier, the N. quadrata Zone should be present between the $N$. lata and $N$. ponticula Zones. Its absence in the cores of Site 959 suggests a hiatus in the lower Miocene sediment. Nannofossil biostratigraphy indicates that nannofossils for the same samples in Hole 959A were reworked, therefore, supporting the presence of a hiatus between the $N$. lata and $N$. ponticula silicoflagellate Zones. Poorly preserved nannofossils of Subzone CN1c co-occur with the $N$. Ponticula silicoflagellate zone, which should not be found in Subzone CN1c; therefore, rare nannofossils from Subzone CN1c are reworked across the hiatus. Nannofossil biostratigraphy also shows that Subzone CN1c is significantly thinner than one would expect given the sedimentation rates of the nannofossil zones above and below Subzone CN1c. The sedimentation rates for Subzones CN1a and CN1b are $72 \mathrm{~m} / \mathrm{m}$.y. and for Zone CN2 the sedimentation rate is $40 \mathrm{~m} / \mathrm{m}$.y., but the sedimentation rate for 
Subzone CN1c is less than $10 \mathrm{~m} / \mathrm{m} . \mathrm{y}$. The relatively low sedimentation rate in Subzone CN1c suggests that a disconformity occurs in the lower Miocene sequence in Subzone CN1c. Works by other authors support this conclusion (Keller and Barron, 1983; Locker and Martini, 1986).

Sites 575 and $591\left(31^{\circ} \mathrm{S}\right.$, in the southwest Pacific) also indicate a missing zone in the lower Miocene rock. At Site 575 in the tropical Pacific, Bukry documents the absence of the N. quadrata Zone and attributes it to a poor silicoflagellate assemblage, not a hiatus, in the cores and also suggests that $N$. quadrata must have a latitudinal preference for the cooler, high-latitude regions (Bukry, 1985). Paleotemperature analyses by Bukry (1985) correlates periods of cool water with hiatus NH1 found by Keller and Barron (1983). Locker and Martini (1986) reviewed cores from Site 206 and recognized a hiatus in the uppermost lower Miocene sequence from nannofossil Zone NN2 through NN4. In the same work they also identified a hiatus in Zones NN2 through NN4 for Site 591. The missing sediments in Sites 591, and the tropical cores of Sites 959 and 575, are likely due to an ocean hiatus (NH1) that occurs in the lower Miocene sediment, from 20 to $18 \mathrm{Ma}$, (Keller and Barron, 1983).

Silicoflagellate relative abundance for the ?lower Oligocene to lower Miocene sediment of Hole 959A (Fig. 2) indicates there were two major pulses of silicoflagellate abundance. The first occurred just before the Oligocene/Miocene boundary in the late Oligocene, and the second pulse of silicoflagellates occurred in the latest early Miocene, just before siliceous sedimentation nearly ceased in the middle Miocene.

The first pulse of abundance is very distinct with a total silicoflagellate count of 1715 specimens. This is good evidence that productivity at this time (latest early Miocene) was high even though the counts were done on smear slides, which may cause a slight distortion of the magnitude of the pulses. The second pulse, near the Oligocene/ Miocene boundary is also distinct with a count of 522 total specimens, but this pulse is more difficult to assign as a time of high productivity because the samples downcore were poorly preserved, showing evidence of dissolution.

At Site 366, in the eastern equatorial Atlantic, productivity was determined using $\delta^{18} \mathrm{O}, \delta^{13} \mathrm{C}$, percent carbonate, and percent biogenic opal (Stein, 1985). Stein's graph of percent biogenic opal at Site 366 shows similar trends for the early Miocene with two periods of biosiliceous preservation. The first period occurred near the Oligocene/ Miocene boundary, and the second occurred in the late early Miocene, which correlates with the data in Figure 2.

By the middle Miocene in the Atlantic Ocean, most biosiliceous sedimentation had abruptly declined. Selected Atlantic Ocean sites in the eastern, western, and southern basins show that a major reorganization in the silica budget had occurred (Mikkelsen and Barron, 1996), causing little or no biosiliceous microfossils to be preserved. This idea of reorganization of the silica budget is not a new one, and previously published works have recognized the change of silica deposition, with silica being deposited mainly in the Atlantic up until the middle Miocene to silica being deposited mainly in the North Pacific after the middle Miocene (Keller and Barron, 1983; Woodruff and Savin, 1989).

\section{CONCLUSIONS}

A silicoflagellate biostratigraphy was constructed for the Oligocene and lower Miocene for Site 959 with five silicoflagellate zones. The zones are the $C$. apiculata Zone, the $N$. biapiculata Zone, the $N$. lata Zone, the $N$. ponticula Zone, and the $C$. triacantha Zone, with Subzone Ds. speculum hemisphaericus occurring in the N. biapiculata Zone. The absence of the $N$. quadrata Zone is indication that a hiatus occurs in the lower Miocene cores of Site 959. The reworked nannofossils in Subzone CN1c and the relatively low sedi- mentation rates in Subzone CN1c support the presence of a hiatus in Subzone CN1c of the lower Miocene cores.

Two siliceous paleoproductivity events punctuated the upper Oligocene and lower Miocene sediments with the first high-productivity event occurring right before the Oligocene/Miocene boundary, and the second high productivity event occurring in the latest early Miocene. Right after the second high-productivity event, siliceous sedimentation comes to an abrupt halt in the Ivorian Basin, supporting a middle Miocene change in distribution of global biogenic silica.

\section{SYSTEMATIC PALEONTOLOGY}

In the systematic paleontology section of this paper the following species were identified using Geological Society of America Memoir 106 (Loeblich et al., 1968): Cannopilus iidaensis, Cannopilus schulzii, and Cannopilus schulzii forma longispinus.

\section{Genus BACHMANNOCENA Locker, 1974, emend. Bukry, 1987}

$$
\text { Bachmannocena apiculata apiculata (Schulz) Bukry }
$$$$
\text { (Pl. 1, Figs. 2, 6) }
$$

Mesocena oamaruensis var. apiculata Schulz, 1928, p. 240, fig. 11. Mesocena apiculata apiculata (Schulz) Bukry, 1975, p. 856, pl. 5, figs. 6, 9. Bachmannocena apiculata apiculata (Schulz) Bukry, 1987, p. 403.

Description: Bachmannocena apiculata apiculata includes all taxa with a three-sided basal ring where the sides of the basal ring were straight to slightly convex. Each of the vertices of the basal ring have a short radial spine.

\section{Bachmannocena apiculata glabra (Schulz) Bukry}

$$
\text { (Pl. 1, Fig. 3) }
$$

Mesocena polymorpha var. triangula Lemmermann fa. glabra Schulz, 1928, p. 237, fig. ?3b, 3c.

Mesocena apiculata glabra (Schulz) Bukry, 1978a, p. 705, pl. 2, figs. 14, 15. Bachmannocena apiculata glabra (Schulz) Bukry, 1987, p. 404.

Description: This species has a three-sided basal ring like B. apiculata apiculata, but the shape of the basal ring is different with two of the sides concave and the third side convex. Three short spines radiate from each vertex.

$$
\text { Bachmannocena apiculata inflata (Bukry) Bukry }
$$

Mesocena apiculata inflata Bukry, 1978c, p. 797, pl. 3, figs. 1-3. Bachmannocena apiculata inflata (Bukry) Bukry, 1987, p. 404.

Description: This taxon has a three-sided basal ring with three short spines at each vertex. It is distinguished from B. apiculata apiculata by having all sides convex so that the basal ring is nearly circular.

$$
\text { Bachmannocena diodon (Ehrenberg) Bukry }
$$

Mesocena diodon Ehrenberg, 1844, p. 84; Martini and Müller, 1976, p. 881, pl. 4 , fig. 4.

Bachmannocena diodon (Ehrenberg) Bukry, 1987, p. 404.

Description: Bachmannocena diodon has an elliptical basal ring with only two spines radiating along the major axis. The skeletal surface may be noded or smooth.

\section{Bachmannocena elliptica (Ehrenberg) Bukry}

$$
\text { (P1. 1, Fig. 1) }
$$

Dictyocha elliptica Ehrenberg, 1840, p. 208

Mesocena elliptica (Ehrenberg) Ehrenberg, 1844, pp. 71, 84. Bachmannocena elliptica (Ehrenberg) Bukry, 1987, p. 404.

Description: Bachmannocena elliptica has a four-sided basal ring that is elliptical, rhomboid, and sometimes circular, with four spines radiating from the vertices. The major axis spines are usually longer than the minor axis spines. This species has a highly variable morphology and it is almost impossible to separate the species into separate groups based on the shape of the bas- 
Table 1. Silicoflagellate abundance data of Hole 959A from the Corbisema triacantha Zone to the Naviculopsis lata Zone.

\begin{tabular}{|c|c|c|c|c|c|c|c|c|c|c|c|c|c|c|c|c|c|c|c|c|c|c|c|c|c|c|}
\hline $\begin{array}{c}\text { Silicoflagellate } \\
\text { zonation }\end{array}$ & Sample & 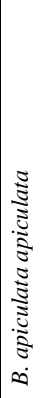 & 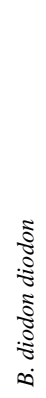 & $\begin{array}{c}\tilde{\Xi} \\
\stackrel{\tilde{\Xi}}{\Xi} \\
\infty \\
\infty\end{array}$ & 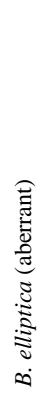 & 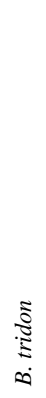 & 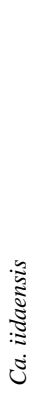 & 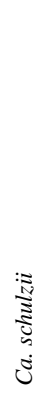 & 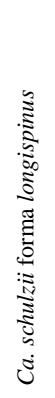 & 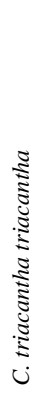 & 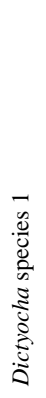 & 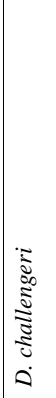 & 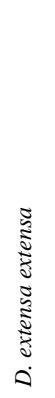 & 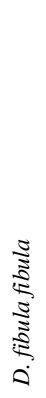 & 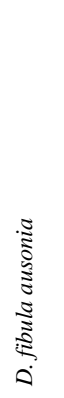 & 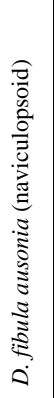 & 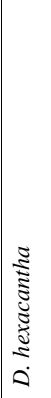 & 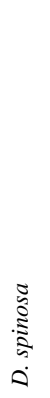 & $\begin{array}{l}\stackrel{\Xi}{\Xi} \\
\vdots \\
0 \\
0\end{array}$ & $\begin{array}{l}\vdots \\
\vdots \\
\dot{0}\end{array}$ & 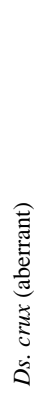 & $\begin{array}{l}\vec{\Xi} \\
\tilde{\Xi} \\
\stackrel{\Xi}{\Xi} \\
\dot{0}\end{array}$ & 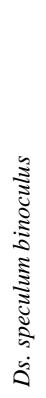 & 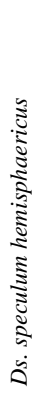 & 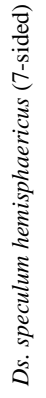 & 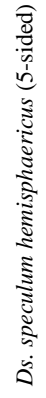 \\
\hline C. triacantha & \begin{tabular}{|l}
$159-959 \mathrm{~A}-$ \\
$23 \mathrm{X}-1,39-41$ \\
$23 \mathrm{X}-4,66-68$ \\
$23 \mathrm{X}-6,89-91$ \\
$23 \mathrm{X}-\mathrm{CC}$ \\
$24 \mathrm{X}-2,59-60$ \\
$24 \mathrm{X}-4,48-51$ \\
$24 \mathrm{X}-6,25-27$ \\
$24 \mathrm{X}-\mathrm{CC}$ \\
$25 \mathrm{X}-4,5-6$ \\
$25 \mathrm{X}-4,145-147$ \\
$25 \mathrm{X}-7,39-41$
\end{tabular} & 14 & & $\begin{array}{r}30 \\
12 \\
1503 \\
108 \\
60 \\
10\end{array}$ & 24 & 2 & 1 & 2 & 1 & $\begin{array}{r}4 \\
57 \\
44 \\
6 \\
\\
\\
36 \\
2 \\
11 \\
\end{array}$ & $\begin{array}{l}1 \\
2 \\
5\end{array}$ & $\begin{array}{l}1 \\
1\end{array}$ & 3 & $\begin{array}{l}1 \\
4\end{array}$ & $\begin{array}{r}1 \\
\\
496 \\
62 \\
23 \\
3 \\
27 \\
160\end{array}$ & $\begin{array}{r}3 \\
217 \\
176 \\
84 \\
2 \\
2\end{array}$ & & & $\begin{array}{r}9 \\
\\
91 \\
3 \\
30 \\
8 \\
20 \\
1 \\
16 \\
8\end{array}$ & $\begin{array}{r}24 \\
33 \\
183 \\
\end{array}$ & $\begin{array}{l}1 \\
3 \\
1 \\
\end{array}$ & 241 & 1 & $\begin{array}{r}25 \\
\\
3 \\
10 \\
1\end{array}$ & & \\
\hline N. ponticula & $\begin{array}{l}25 X-C C \\
26 X-3,116-117 \\
26 X-5,37-38 \\
26 X-C C \\
27 X-3,37-38 \\
27 X-5,23-25 \\
27 X-C C \\
28 X-3,9-11 \\
28 X-4,59-61 \\
28 X-6,106-108 \\
28 X-C C \\
29 X-1,13-15 \\
29 X-3,38-40\end{array}$ & 1 & 1 & $\begin{array}{r}22 \\
16 \\
27 \\
30 \\
8 \\
29 \\
1 \\
8 \\
2\end{array}$ & & 1 & & & & $\begin{array}{r}10 \\
15 \\
\\
14 \\
\\
4 \\
2 \\
1 \\
5\end{array}$ & $\begin{array}{l}2 \\
2 \\
1 \\
1 \\
1\end{array}$ & & & & & & $\begin{array}{l}1 \\
\\
\\
1\end{array}$ & 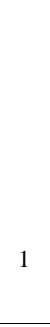 & 1 & $\begin{array}{r}24 \\
12 \\
21 \\
5 \\
14 \\
18 \\
48 \\
56 \\
4 \\
78 \\
3 \\
81 \\
44 \\
\end{array}$ & $\begin{array}{l}2 \\
3\end{array}$ & $\begin{array}{r}110 \\
10 \\
3 \\
5 \\
10 \\
25 \\
17 \\
\\
\\
6\end{array}$ & $\begin{array}{l}1 \\
7\end{array}$ & $\begin{array}{r}2 \\
\\
36 \\
4 \\
6 \\
3\end{array}$ & & \\
\hline N. lata & $\begin{array}{l}\text { 29X-5, 30-33 } \\
\text { 29X-CC } \\
\text { 30X-1, 30-32 } \\
30 X-3,86-88 \\
30 X-5,86-88 \\
\text { 30X-7, 36-38 } \\
\text { 30X-CC } \\
31 X-1,24-25 \\
31 X-2,128-130 \\
31 X-5,67-68\end{array}$ & & & 3 & & & & & & 2 & & & & $\begin{array}{l}2 \\
4\end{array}$ & $\begin{array}{r}2 \\
6 \\
12 \\
4 \\
4 \\
13 \\
8 \\
\\
2\end{array}$ & 1 & & & & $\begin{array}{r}34 \\
11 \\
3 \\
9 \\
8 \\
13 \\
3 \\
4 \\
12 \\
11\end{array}$ & 9 & & & $\begin{array}{r}3 \\
11 \\
3\end{array}$ & 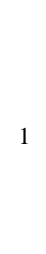 & 1 \\
\hline
\end{tabular}

al ring. Thus rhomboid, elliptical, and circular skeletons were grouped together as B. elliptica.

Remarks: Bachmannocena elliptica occurs in Samples 159-959A-29X-5, 30-33 cm, through 159-959A-27X-3, 37-38 cm, and Samples 159-959A$24 \mathrm{X}-4,48-51 \mathrm{~cm}$, through 159-959A-23X-1, 39-41 cm, intervals from the lower Miocene sequence. Several authors (Bukry, 1980; Bukry, 1978b; McCartney et al., 1995) make the distinction between B. elliptica and B. quadrata with the Quaternary $B$. quadrata having a more noded surface and a more quadrate form than the lower Miocene B. elliptica. In these cores, this taxon has a varied morphology ranging from small, elliptical, and noded to large, quadrate, and delicate. Because of the variation in morphology of this species, all of the forms were grouped into one species, B. elliptica. In Sample 159959A-23X-6, 89-91 cm, 1503 specimens of B. elliptica were counted. With such a large count of $B$. elliptica it is possible that nutrient supply to the surface water at this time was very high and caused a wide range of skeletal morphologies in B. elliptica species, often times very robust and ornate.

\section{Bachmannocena triodon (Bukry) Bukry}

$$
\text { (Pl. 1, Fig. 11) }
$$

Mesocena triodon Bukry, 1973, p. 860, pl. 2, fig. 11; Bukry, 1978b, p. 839, pl. 7, figs. 9, 10; McCartney and Wise, 1987, p. 813, pl. 4, figs. 1-4. Bachmannocena triodon (Bukry) Bukry, 1987, p. 405.

Description: Bachmannocena triodon has an elliptical basal ring and three spines. Two of the spines radiate from the major axis and the third spine occurs on one side of the minor axis. Only three specimens were counted from
Site 959, two specimens occurred in Sample 159-959A-23X-6, 89-91 cm, and one specimen occurred in Sample 159-959A-28X-6, 106-108 cm.

Genus CANNOPILUS Haeckel, 1887

Cannopilus iidaensis Bachmann

Cannopilus iidaensis Bachmann in Ichikawa and others 1967, pl. 52, figs. 1-7; Locker and Martini, 1986, p. 912, pl. 1, figs. 5, 6.

Description: The basal ring of this taxon has many basal spines radiating at about the same length with the number of spines varying. In the description given by Locker and Martini, the basal ring has nine spines, but the preservation of these specimens in Hole 959A was so poor that the number of basal spines was not evident. The cannopolid silicoflagellates were commonly fragmented and broken whereas other silicoflagellates were well preserved; this may be due to their larger size. The apical structure is cannopolid (elevated and spherical) and has many irregularly shaped apical windows.

\section{Cannopilus schulzii (Deflandre) Schulz}

Cannopilus schulzii Deflandre in Bachmann and Ichikawa 1962; Cannopilus schulzii (Deflandre), Perch-Nielsen, 1985, p. 824, pl. 9. fig. 4.

Description: This taxon has a hemispherical apical structure with a varying number of apical windows. The basal ring is eight sided with eight radiating spines. 
Table 1 (continued).

\begin{tabular}{|c|c|c|c|c|c|c|c|c|c|c|c|c|c|c|c|c|c|c|c|c|}
\hline $\begin{array}{l}\text { Silicoflagellate } \\
\text { zonation }\end{array}$ & Sample & 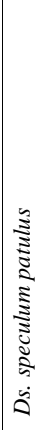 & 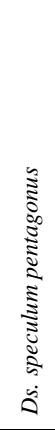 & 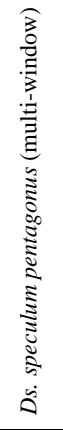 & 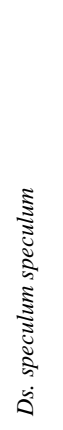 & 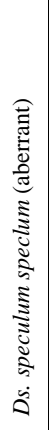 & 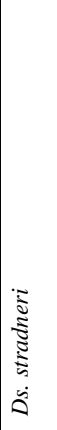 & 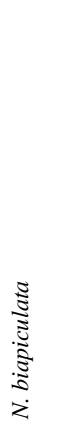 & 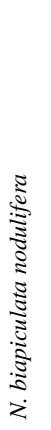 & 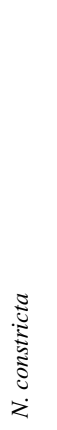 & 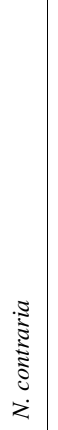 & $\frac{\frac{\Xi}{3}}{z}$ & 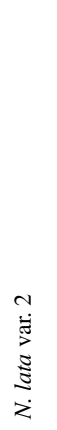 & 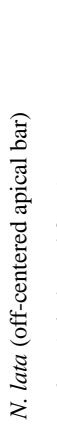 & 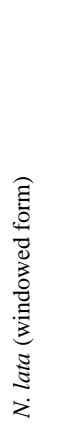 & 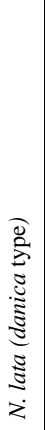 & 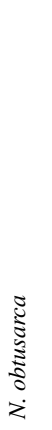 & 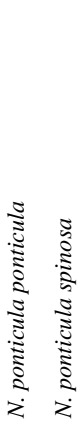 & 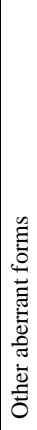 & 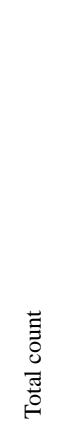 \\
\hline C. triacantha & \begin{tabular}{|l}
$159-959 A-$ \\
$23 X-1,39-41$ \\
$23 X-4,66-68$ \\
$23 X-6,89-91$ \\
$23 X-C C$ \\
$24 X-2,59-60$ \\
$24 X-4,48-51$ \\
$24 X-6,25-27$ \\
$24 X-C C$ \\
$25 X-4,5-6$ \\
$25 X-4,145-147$ \\
$25 X-7,39-41$ \\
\end{tabular} & $\begin{array}{l}1 \\
2\end{array}$ & 10 & & $\begin{array}{l}1 \\
1\end{array}$ & 1 & & & & 1 & & & & & & & & & 4 & $\begin{array}{r}44 \\
13 \\
1715 \\
111 \\
867 \\
273 \\
128 \\
6 \\
108 \\
215 \\
469 \\
\end{array}$ \\
\hline N. ponticula & $\begin{array}{l}\text { 25X-CC } \\
26 \mathrm{X}-3,116-117 \\
26 \mathrm{X}-5,37-38 \\
26 \mathrm{X}-\mathrm{CC} \\
27 \mathrm{X}-3,37-38 \\
27 \mathrm{X}-5,23-25 \\
27 \mathrm{X}-\mathrm{CC} \\
28 \mathrm{X}-3,9-11 \\
28 \mathrm{X}-4,59-61 \\
28 \mathrm{X}-6,106-108 \\
28 \mathrm{X}-\mathrm{CC} \\
29 \mathrm{X}-1,13-15 \\
29 \mathrm{X}-3,38-40\end{array}$ & $\begin{array}{l}5 \\
2\end{array}$ & $\begin{array}{r}2 \\
1 \\
1 \\
12\end{array}$ & & 4 & 1 & $\begin{array}{r}1 \\
1 \\
3 \\
\\
6 \\
14 \\
2 \\
2\end{array}$ & & & $\begin{array}{l}3 \\
6\end{array}$ & & $\begin{array}{l}8 \\
3\end{array}$ & & & & & $\begin{array}{r}2 \\
\\
1 \\
28 \\
2\end{array}$ & $\begin{array}{rrr}1 & & \\
1 & \\
2 & \\
& 6 \\
& 1 \\
& 1 \\
& \\
3 & \\
1 & \\
14 & \\
2 & \end{array}$ & 1 & $\begin{array}{r}155 \\
49 \\
29 \\
17 \\
132 \\
77 \\
118 \\
108 \\
24 \\
163 \\
6 \\
106 \\
48\end{array}$ \\
\hline N. lata & $\begin{array}{l}29 X-5,30-33 \\
\text { 29X-CC } \\
\text { 30X-1, 30-32 } \\
\text { 30X-3, 86-88 } \\
\text { 30X-5, 86-88 } \\
\text { 30X-7, 36-38 } \\
\text { 30X-CC } \\
\text { 31X-1, 24-25 } \\
\text { 31X-2, 128-130 } \\
\text { 31X-5, 67-68 }\end{array}$ & & 1 & 1 & $\begin{array}{l}3 \\
2 \\
2 \\
2\end{array}$ & & & $\begin{array}{r}4 \\
1 \\
122 \\
31\end{array}$ & 2 & $\begin{array}{l}1 \\
1 \\
3\end{array}$ & & $\begin{array}{r}16 \\
40 \\
29 \\
23 \\
147 \\
197 \\
37 \\
19 \\
2 \\
46\end{array}$ & $\begin{array}{r} \\
\\
\\
\\
60 \\
71 \\
15 \\
1 \\
24\end{array}$ & $\begin{array}{r}14 \\
4 \\
2 \\
3\end{array}$ & $\begin{array}{r}1 \\
13 \\
2 \\
5\end{array}$ & 2 & & & 1 & $\begin{array}{r}59 \\
58 \\
46 \\
32 \\
167 \\
337 \\
127 \\
60 \\
141 \\
125\end{array}$ \\
\hline
\end{tabular}

Cannopilus schulzii forma longispinus Bachmann and Ichikawa

Cannopilus schulzii forma longispinus Bachmann and Ichikawa, 1962; Loeblich et al., 1968, p. 205, pl. 2, figs. 6-24.

\section{Genus CORBISEMA Hanna, 1928}

\section{Corbisema apiculata (Lemmermann) Hanna}

$$
\text { (Pl. 1, Fig. 7) }
$$

Dictyocha triacantha var. apiculata Lemmermann, 1901, p. 259, pl. 10, figs. 19, 20.

Corbisema apiculata (Lemmermann) Hanna, 1931, p. 187-201; PerchNielsen, 1975, p. 685, pl. 2, figs. 15, 16, 19.

\section{Corbisema apiculata var. 1}

Description: This taxon is similar to $C$. apiculata in its size, but the basal spines are longer than those of $C$. apiculata and the skeleton is more robust and noded.

\section{Corbisema hastata globulata Bukry}

Corbisema hastata globulata Bukry, 1976a, p. 907, pl. 4, figs. 1-8.

Corbisema triacantha mediana Bukry

(P1. 1, Fig. 12)
Corbisema triacantha mediana Bukry, 1978a, p. 703, pl. 1, figs. 8-12.

Corbisema triacantha triacantha (Ehrenberg) Bukry

(P1. 1, Fig. 5)

Dictyocha triacantha Ehrenberg, 1844, p. 80.

Corbisema triacantha triacantha (Ehrenberg) Bukry, 1978a, p. 703, pl. 1, figs. $13-15$.

Description: This is a three-sided species with three spines radiating from the vertices and three apical bars that meet at the center of the skeleton. This species has a variable morphology; the corners of the basal ring may be rounded to slightly angular, and the length of the spines is variable from short to medium.

\section{Corbisema triacantha var. 1}

$$
\text { (P1. 1, Fig. 8) }
$$

Description: These specimens include those that had a basal ring smaller and more convex than that of $C$. triacantha triacantha. The basal ring of $C$. triacantha triacantha is commonly indented where the apical struts meet the basal ring; whereas, specimens designated Corbisema triacantha variety 1 do not have that indention. Corbisema triacantha variety 1 also has a basal ring comparable to D. spinosa in its shape, but larger. 
Table 2. Silicoflagellate abundance data of Hole 959A in the Naviculopsis biapiculata Zone.

\begin{tabular}{|c|c|c|c|c|c|c|c|c|c|c|c|c|c|c|c|c|c|c|c|c|}
\hline \multicolumn{2}{|l|}{$\begin{array}{l}\text { Silicoflagellate } \\
\text { zonation }\end{array}$} & Sample & 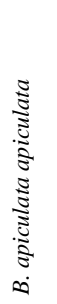 & 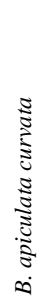 & 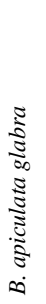 & 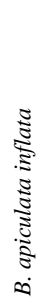 & 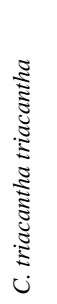 & 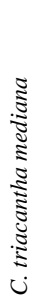 & 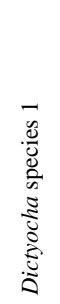 & 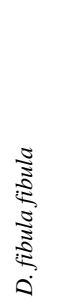 & 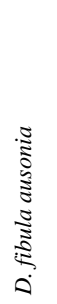 & 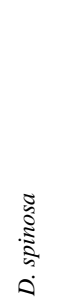 & $\begin{array}{l}\vec{u} \\
\mathbf{E} \\
\dot{0} \\
\dot{0}\end{array}$ & 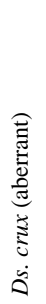 & 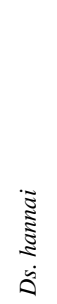 & 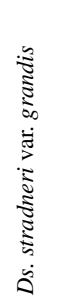 & 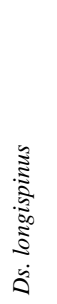 & 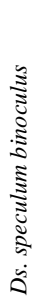 & 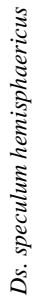 & 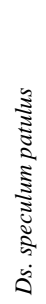 \\
\hline & $\begin{array}{l}\text { Ds. speculum } \\
\text { hemisphaericus }\end{array}$ & $\begin{array}{l}\text { 159-959A- } \\
\text { 31X-7, 2-3 } \\
\text { 31X-CC } \\
\text { 32X-1, 49-51 } \\
\text { 32X-3, 98-100 } \\
\text { 32X-5, 40-41 } \\
\text { 32X-CC } \\
\text { 33X-2, 149-150 } \\
\text { 33X-4, 58-59 } \\
\text { 33X-6, 62-64 } \\
\text { 33X-CC } \\
\text { 34X-2, 64-65 } \\
\text { 34X-4, 72-74 } \\
\text { 34X-6, 44-45 } \\
\text { 34X-CC } \\
\text { 35X-1, 93-94 } \\
\text { 35X-3, 59-61 } \\
\text { 35X-5, 8-10 } \\
\text { 35X-CC } \\
\text { 36X-1, 109-110 } \\
\text { 36X-3, 88-89 } \\
\text { 36X-5, 46-48 } \\
\text { 36X-CC } \\
\text { 37X-1, 1-3 } \\
\text { 37X-3, 51-52 } \\
\text { 37X-6, 0-1 } \\
\text { 37X-CC } \\
\text { 38X-1, 31-33 } \\
\text { 38X-3, 100-102 } \\
\text { 38X-5, 71-73 } \\
\text { 38X-CC } \\
\text { 39X-1, 13-14 } \\
\text { 39X-3, 58-59 } \\
\text { 39X-5, 123-124 }\end{array}$ & $\begin{array}{r}6 \\
18 \\
16 \\
10 \\
20 \\
16 \\
1 \\
2 \\
7 \\
10 \\
45 \\
5 \\
2 \\
2 \\
\\
9 \\
16 \\
7 \\
48 \\
18 \\
11 \\
25 \\
2 \\
8 \\
8 \\
24 \\
27 \\
31\end{array}$ & 2 & $\begin{array}{l}1 \\
6 \\
2 \\
2 \\
1 \\
1\end{array}$ & $\begin{array}{l}1 \\
1 \\
1 \\
\\
1\end{array}$ & $\begin{array}{r} \\
\\
\\
\\
\\
\\
\\
4 \\
3 \\
18 \\
19\end{array}$ & 1 & $\begin{array}{r}15 \\
1 \\
1 \\
3 \\
1 \\
1 \\
1 \\
1\end{array}$ & $\begin{array}{l}1 \\
2 \\
1 \\
5\end{array}$ & $\begin{array}{r}9 \\
25 \\
13 \\
44 \\
1 \\
26 \\
3\end{array}$ & 1 & $\begin{array}{r}4 \\
53 \\
32 \\
25 \\
77 \\
13 \\
104 \\
23 \\
9 \\
31 \\
6 \\
28 \\
43 \\
106 \\
16 \\
1 \\
\\
\\
3 \\
1 \\
3 \\
2\end{array}$ & $\begin{array}{l}1 \\
2 \\
3 \\
1\end{array}$ & $\begin{array}{r}131 \\
70 \\
76 \\
16 \\
4 \\
4 \\
1 \\
1 \\
3 \\
\end{array}$ & $\begin{array}{r}3 \\
13 \\
5 \\
35\end{array}$ & $\begin{array}{r}1 \\
8 \\
20 \\
1 \\
17 \\
15 \\
29 \\
87\end{array}$ & 2 & $\begin{array}{l}1 \\
1 \\
1 \\
1 \\
4\end{array}$ & 3 \\
\hline N. biapiculata & & $\begin{array}{l}39 \mathrm{X}-\mathrm{CC} \\
40 \mathrm{X}-1,63-65 \\
40 \mathrm{X}-4,93-95 \\
40 \mathrm{X}-5,50-52 \\
40 \mathrm{X}-\mathrm{CC} \\
41 \mathrm{X}-1,22-23\end{array}$ & $\begin{array}{l}6 \\
1\end{array}$ & & & & $\begin{array}{l}6 \\
2\end{array}$ & & & & & & & & & & & & & \\
\hline
\end{tabular}

\section{Genus DICTYOCHA Ehrenberg}

Dictyocha species 1

(Pl. 2, Fig. 4)

Description: Specimens of Dictyocha sp. cf. Ds. crux include forms that have basal rings similar to the basal ring of Ds. crux, and the same variance of spine length of Ds. crux. The only difference is that Dictyocha sp. cf. Ds. crux has an apical bar structure instead of an apical window that may be oriented parallel to the major or minor axis.

\section{Dictyocha challengeri Martini and Müller}

Dictyocha challengeri Martini and Müller, 1976, p. 878, pl. 2, fig. 8; pl. 8, fig. 3; Shaw and Ciesielski, 1983, p. 722, pl. 5, figs. 5, 10.

\section{Dictyocha deflandrei Frenguelli ex Glezer} (Pl. 1, Fig. 13)

Dictyocha deflandrei Frenguelli, 1940 (in part), p. 65, fig. 14a, c, d, ?e, and f; Bukry (in part), 1975, p. 863, pl. 2, figs. 11, 12; Perch-Nielsen, 1985, p. 828 , pl. 15, figs. 5-7.

\section{Dictyocha extensa extensa (Locker) Locker and Martini}

(Pl. 1, Figs. 10, 15)

Dictyocha varia f. extensa Locker, 1975, pp. 99-101, figs. 1/2, 3/3.
Dictyocha extensa (Locker) Locker and Martini, 1986, pp. 903-904, pl. 2, figs. $10-12$; pl. 11, fig. 3.

Dictyocha extensa extensa (Locker) Locker and Martini, McCartney et al., 1995, pl. 3, figs. 2-5; pl. 8, fig. 8 .

Description: Dictyocha extensa extensa includes all specimens with a basal ring identical or similar to $D$. varia, with the apical bar oriented along the major axis, unlike $D$. varia, which has an apical bar oriented along the minor axis. In these samples $D$. extensa extensa is very rare, occurring only three times, all in Sample 159-959A-23X-6, 89-91 cm.

Dictyocha fibula ausonia (Deflandre) McCartney et al.

(Pl. 1, Fig. 14)

Dictyocha ausonia Deflandre, 1950, p. 195, figs. 194-196, 199-202; Ling (in part), 1972, p. 216, pl. 25, figs. 1-5, 9, 10 (not 6-8).

Dictyocha brevispina ausonia (Deflandre) Bukry, 1978a, p. 703, pl. 1, figs. 17-19.

Dictyocha fibula ausonia (Deflandre) McCartney et al., 1995, pl. 2, fig. 2; pl. 8, fig. 6 .

Description: This taxon has an apical bar along the minor axis with four spines from each of the vertices. There are two portals where the apical struts meet the basal ring.

Remarks: McCartney et al., 1995, changed the name of this taxon and several others that have been previously attributed as aspera or fibula, depend- 
Table 2 (continued).

\begin{tabular}{|c|c|c|c|c|c|c|c|c|c|c|c|c|c|c|c|c|c|}
\hline \multicolumn{2}{|l|}{$\begin{array}{l}\text { Silicoflagellate } \\
\text { zonation }\end{array}$} & Sample & 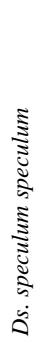 & 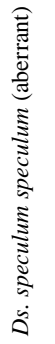 & 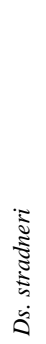 & 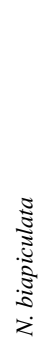 & 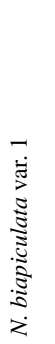 & 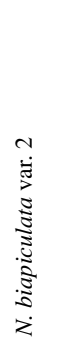 & 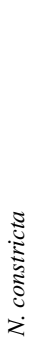 & 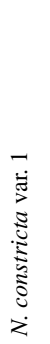 & 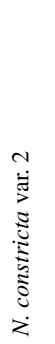 & 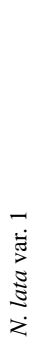 & 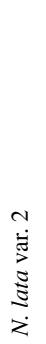 & 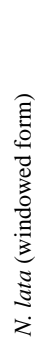 & 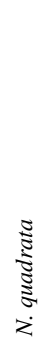 & 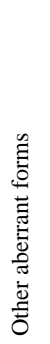 & 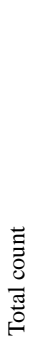 \\
\hline & $\begin{array}{l} \\
\\
\text { Ds. speculum } \\
\text { hemisphaericus }\end{array}$ & 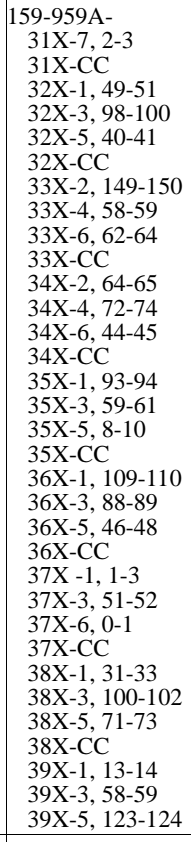 & $\begin{array}{r}2 \\
1 \\
1 \\
10 \\
34 \\
42 \\
\\
8 \\
\\
1 \\
28 \\
\\
\\
1 \\
2 \\
7 \\
1 \\
26 \\
22 \\
2 \\
12 \\
15 \\
4 \\
1 \\
2 \\
1 \\
2 \\
2 \\
\end{array}$ & 4 & 1 & $\begin{array}{r}17 \\
3\end{array}$ & $\begin{array}{r}25 \\
6 \\
\\
3\end{array}$ & $\begin{array}{r}2 \\
5 \\
9 \\
8 \\
1 \\
3 \\
11 \\
17 \\
27 \\
3 \\
1 \\
64 \\
3 \\
159\end{array}$ & $\begin{array}{l}1 \\
2 \\
2 \\
4 \\
1\end{array}$ & $\begin{array}{l}7 \\
1\end{array}$ & $\begin{array}{r}38 \\
18 \\
1\end{array}$ & $\begin{array}{r}2 \\
36 \\
48\end{array}$ & 1 & 1 & 1 & $\begin{array}{l}1 \\
\\
4 \\
2 \\
6 \\
1 \\
1 \\
1 \\
2 \\
1 \\
10\end{array}$ & $\begin{array}{r}78 \\
146 \\
87 \\
96 \\
122 \\
74 \\
217 \\
277 \\
109 \\
125 \\
51 \\
133 \\
134 \\
522 \\
21 \\
4 \\
5 \\
1 \\
5 \\
35 \\
22 \\
41 \\
78 \\
29 \\
37 \\
43 \\
7 \\
2 \\
36 \\
2 \\
34 \\
49 \\
58 \\
\end{array}$ \\
\hline N. biapiculata & & $\begin{array}{l}39 \mathrm{X}-\mathrm{CC} \\
40 \mathrm{X}-1,63-65 \\
40 \mathrm{X}-4,93-95 \\
40 \mathrm{X}-5,50-52 \\
40 \mathrm{X}-\mathrm{CC} \\
41 \mathrm{X}-1,22-23\end{array}$ & & & & & & $\begin{array}{l}1 \\
1\end{array}$ & 1 & & & 4 & & & & & $\begin{array}{r}2 \\
0 \\
18 \\
3 \\
0 \\
0\end{array}$ \\
\hline
\end{tabular}

ing on the orientation of the apical bar. The author accepts McCartney's more recent name designations.

Dictyocha fibula ausonia (Deflandre) (naviculopsid) McCartney et al. (P1. 2, Fig. 1)

Dictyocha sp. cf. Dictyocha brevispina ausonia (Deflandre) Bukry, 1980, p. 513, pl. 1, figs. 7-14.

Dictyocha fibula ausonia (Deflandre) (naviculopsid) McCartney et al., 1995, pl. 2 , fig. 4 .

Description: This taxon has an elliptical basal ring with four spines radiating from the major and minor axes. It also has an off-centered apical bar parallel to the minor axis. It differs from $D$. brevispina ausonia in that the apical bar is displaced from the center of the skeleton and that there are no portals and struts in the apical structure.

\section{Dictyocha fibula fibula Ehrenberg} (Pl. 1, Fig. 9)

Dictyocha fibula Ehrenberg, Locker, 1974, p. 636, pl. 1, fig. 6 (=lectotype). Dictyocha fibula fibula Ehrenberg, Locker and Martini, 1986, p. 904, pl. 5, figs. 1,2 ; pl. 11, figs. 8, 9 .
Dictyocha fibula fibula Ehrenberg, McCartney et al., 1995, pl. 2, fig. 1; pl. 5, fig. 5.

Dictyocha hexacantha Schulz

Dictyocha hexacantha Schulz, 1928, p. 255, fig. 43; Martini and Müller, 1976 , p. 878 , pl. 2., fig. 7 ; p. 886 , pl. 7 , fig. 10.

Remarks: Eocene Dictyocha hexacantha was found in two cores of the early Miocene, which is younger than its known biostratigraphic range. Lithologic data indicate that the samples in which this taxon are present are moderately to heavily bioturbated, suggesting possible reworking.

Dictyocha spinosa (Deflandre) Glezer (P1. 1, Fig. 4)

Corbisema spinosa Deflandre, 1950, p. 193, figs. 178-182.

Dictyocha spinosa (Deflandre) Glezer, 1966, p. 238, pl. 10, figs. 6-8; McCartney and Wise, 1987, p. 806, pl. 1, fig. 6.

Remarks: Eocene Dictyocha spinosa was found in four samples from Hole 959A, the first three specimens were found in core catchers of the upper Oligocene and lower Miocene cores, and the fourth specimen was found in 
Table 3. Silicoflagellate abundance data of Hole 959A from the Naviculopsis biapiculata Zone to the Corbisema apiculata Zone.

\begin{tabular}{|c|c|c|c|c|c|c|c|c|c|c|c|c|c|c|c|c|c|c|c|c|}
\hline $\begin{array}{l}\text { Silicoflagellate } \\
\text { zonation }\end{array}$ & Sample & 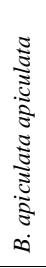 & 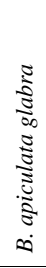 & 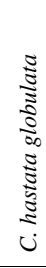 & 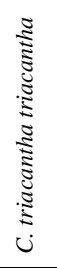 & 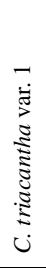 & 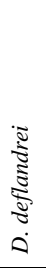 & 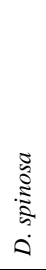 & 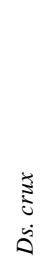 & 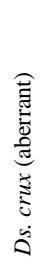 & 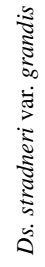 & 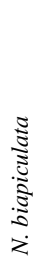 & 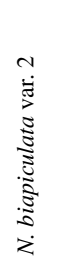 & 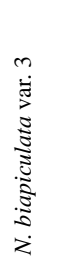 & 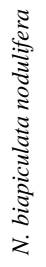 & 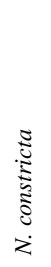 & $\begin{array}{l}7 \\
\overrightarrow{0} \\
\bar{z} \\
\bar{\Xi} \\
\dot{z}\end{array}$ & 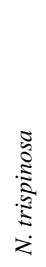 & 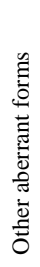 & 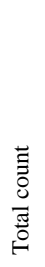 \\
\hline N. biapiculata & \begin{tabular}{|} 
159-959A- \\
$41 \mathrm{X}-4,48-50$ \\
$41 \mathrm{X}-6,11-13$ \\
$41 \mathrm{X}-\mathrm{CC}$ \\
$42 \mathrm{X}-2,60-62$ \\
$42 \mathrm{X}-4,50-53$ \\
$42 \mathrm{X}-7,17-19$
\end{tabular} & $\begin{array}{l}2 \\
1 \\
3 \\
1 \\
2 \\
\end{array}$ & & & $\begin{array}{l}2 \\
1 \\
4 \\
4 \\
7\end{array}$ & & & & 2 & & $\begin{array}{l}1 \\
1\end{array}$ & 1 & 1 & 1 & & 2 & 1 & & & $\begin{array}{r}4 \\
4 \\
6 \\
9 \\
1 \\
13 \\
\end{array}$ \\
\hline C. apiculata & $\begin{array}{l}\text { 42X-CC } \\
43 \mathrm{X}-2,91-92 \\
43 \mathrm{X}-4,45-47 \\
43 \mathrm{X}-6,54-56 \\
43 \mathrm{X}-\mathrm{CC} \\
44 \mathrm{X}-1,44-45 \\
44 \mathrm{X}-3,5-6 \\
44 \mathrm{X}-5,35-37 \\
44 \mathrm{X}-\mathrm{CC} \\
45 \mathrm{X}-1,110-111 \\
45 \mathrm{X}-3,101-102 \\
45 \mathrm{X}-5,120-121 \\
45 \mathrm{X}-\mathrm{CC} \\
46 \mathrm{X}-1,21-23 \\
46 \mathrm{X}-4,7-9 \\
46 \mathrm{X}-7,25-27 \\
\text { 46X-CC }\end{array}$ & $\begin{array}{r}1 \\
6 \\
7 \\
1 \\
1 \\
2 \\
5 \\
11 \\
1 \\
15 \\
2\end{array}$ & 3 & 2 & $\begin{array}{r}1 \\
7 \\
9 \\
11 \\
14 \\
13 \\
4 \\
2\end{array}$ & $\begin{array}{r}15 \\
8 \\
2 \\
1 \\
1\end{array}$ & $\begin{array}{r}5 \\
1 \\
1 \\
11\end{array}$ & 1 & $\begin{array}{l}2 \\
2\end{array}$ & 1 & 1 & $\begin{array}{r}30 \\
29 \\
17 \\
18 \\
7 \\
47 \\
5 \\
6 \\
3 \\
25 \\
1\end{array}$ & $\begin{array}{l}1 \\
1 \\
2 \\
2\end{array}$ & $\begin{array}{r}3 \\
2 \\
2 \\
4 \\
14 \\
3 \\
7 \\
6 \\
3 \\
\end{array}$ & 4 & $\begin{array}{l}3 \\
2\end{array}$ & $\begin{array}{r}16 \\
3 \\
\\
1\end{array}$ & $\begin{array}{r}2 \\
10\end{array}$ & $\begin{array}{l}1 \\
1 \\
1\end{array}$ & $\begin{array}{r}8 \\
53 \\
77 \\
28 \\
19 \\
15 \\
76 \\
39 \\
36 \\
20 \\
67 \\
13 \\
17 \\
19 \\
4 \\
0 \\
7\end{array}$ \\
\hline
\end{tabular}

Table 4. Silicoflagellate abundance data of Hole 959D in the Corbisema apiculata Zone.

\begin{tabular}{|c|c|c|c|c|c|c|c|c|c|c|c|c|c|c|c|c|c|c|c|}
\hline $\begin{array}{l}\text { Silicoflagellate } \\
\text { zonation }\end{array}$ & Sample & 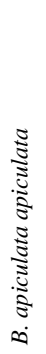 & 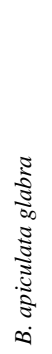 & 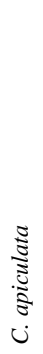 & 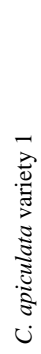 & 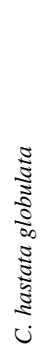 & 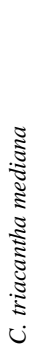 & 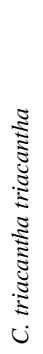 & 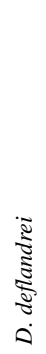 & 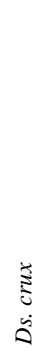 & 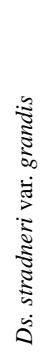 & 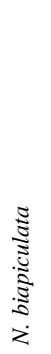 & 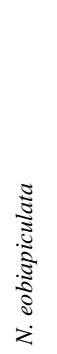 & 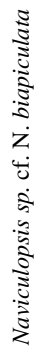 & 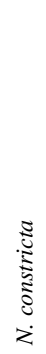 & 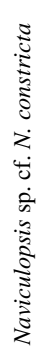 & 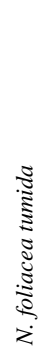 & 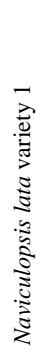 & 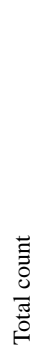 \\
\hline C. apiculata & $\begin{array}{l}\text { 159-959D- } \\
\text { 1R-CC } \\
\text { 2R-1, 14-15 } \\
\text { 2R-CC } \\
\text { 3R-CC, 9-10 } \\
\text { 3R-CC } \\
\text { 4R-1, 8-9 } \\
\text { 4R-CC } \\
\text { 5R-CC, 1-2 } \\
\text { 5R-CC } \\
\text { 6R-CC, 1-2 } \\
\text { 6R-CC } \\
\text { 7R-CC }\end{array}$ & $\begin{array}{l}1 \\
1 \\
1 \\
5\end{array}$ & 1 & 1 & $\begin{array}{l}1 \\
1 \\
1\end{array}$ & $\begin{array}{l}6 \\
1 \\
1 \\
2\end{array}$ & 2 & $\begin{array}{l}4 \\
5 \\
4 \\
1 \\
2\end{array}$ & $\begin{array}{l}1 \\
1\end{array}$ & $\begin{array}{l}3 \\
1\end{array}$ & 1 & $\begin{array}{l}1 \\
1\end{array}$ & $\begin{array}{l}2 \\
1\end{array}$ & $\begin{array}{l}4 \\
4\end{array}$ & $\begin{array}{l}12 \\
35\end{array}$ & 2 & 1 & 2 & $\begin{array}{r}19 \\
9 \\
7 \\
5 \\
5 \\
0 \\
0 \\
29 \\
54 \\
0 \\
0 \\
30\end{array}$ \\
\hline
\end{tabular}

Sample 159-959A-44X-3, 5-6 cm, in the C. apiculata Zone (?lower Oligocene, ?upper Eocene). The sediments in which D. spinosa are present are moderately to heavily bioturbated, suggesting possible reworking.

\section{Dictyocha varia Locker}

(Pl. 2, Figs. 2, 3)

Dictyocha varia Locker, 1975, pp. 99-101, figs. 3-7.

Dictyocha pulchella Bukry, 1975, pp. 677-701.

Dictyocha varia Locker, McCartney et al., 1995, pl. 5, fig. 2 (not pl. 8, fig. 6).

Genus DISTEPHANUS Stohr

Distephanus crux Ehrenberg

(Pl. 2, Figs. 5, 6)
Distephanus crux Ehrenberg, 1840, p. 207; McCartney and Wise, 1987, p. 811, pl. 2, figs. 10-11.

Distephanus crux crux (Ehrenberg) Bukry, 1979b, p. 999, pl. 5, fig. 2; McCartney et al., 1995, p. 159, pl. 7, fig. 9 .

Distephanus crux carolae (Ehrenberg) Bukry (in part), 1982, p. 322, pl. 5. figs. 5-10 (not 4, 11).

Distephanus crux scutulatus (Ehrenberg) Bukry, 1985, p. 494, pl. 3, figs. 7, 8.

Description: This is a four-sided Distephanus with a square to round apical opening. The shape of the basal ring can be round or square, with no indention where the apical struts meet the basal ring. The spine lengths are short with the longer spines on the major axis. The apical opening for Ds. crux is larger than the apical opening of Ds. hannai, also the basal ring for Ds. hannai is indented where the struts meet the basal ring, and more diamond shaped. This taxon is commonly smaller than Ds. hannai. 


\section{Silicoflagellate Abundance}

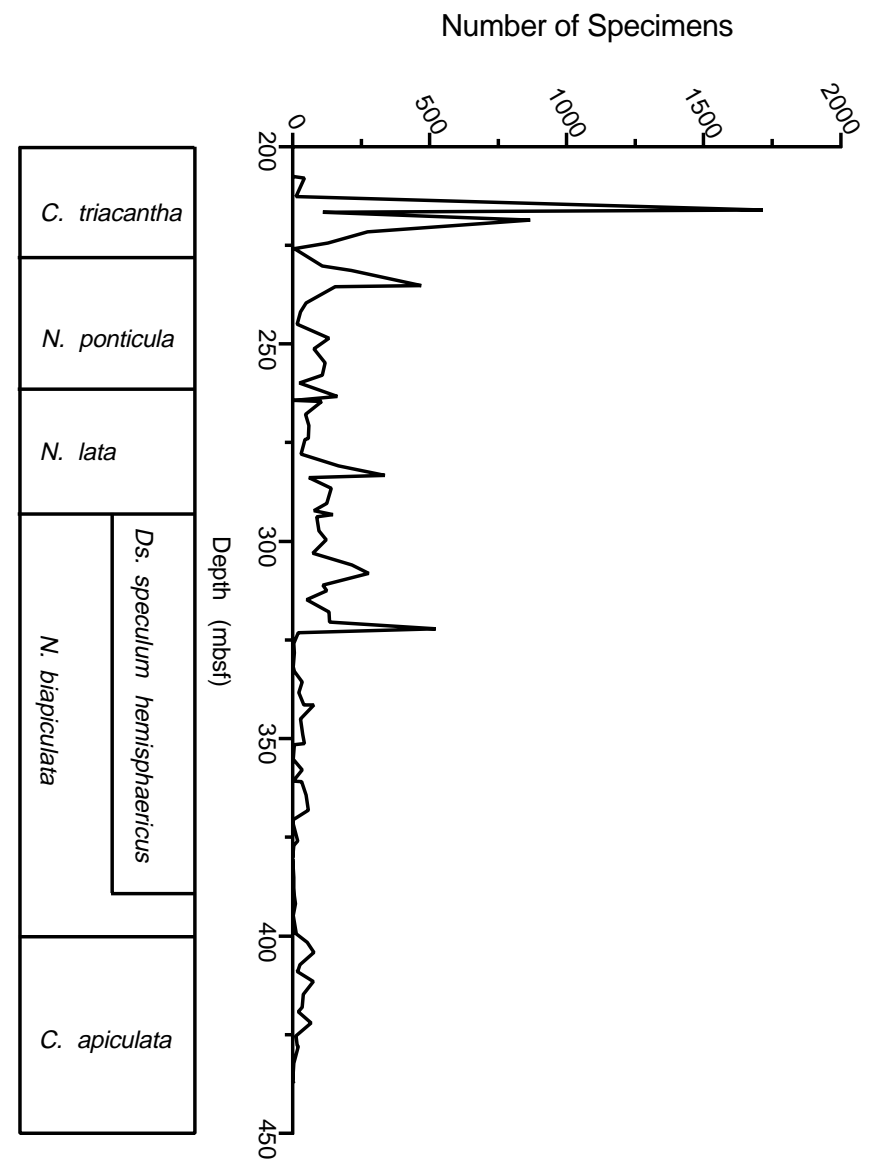

Figure 2. Graph of silicoflagellate abundance vs. age for Hole 959A, with a hiatus occurring between the Naviculopsis lata and Naviculopsis ponticula Zones of the lower Miocene cores.

Remarks: This is a broad designation for Distephanus species with a four-sided apical window, four spines, and a four-sided basal ring, and includes all species and subspecies listed above.

Distephanus hannai (Bukry) Bukry

Distephanus crux hannai Bukry, 1975, p. 867, pl. 4., figs. 4-6. Distephanus hannai (Bukry) Bukry, 1979a, p. 561.

Description: This taxon is a four-sided Distephanus with four spines radiating from the vertices of the basal ring. The spines along the major axis are longer than the spines along the minor axis. The apical opening is round to square and is smaller than the apical opening for Ds. crux. Distephanus hannai also has a basal ring that is distinguished from Ds. crux by having more indention where the basal ring and struts meet.

\section{Distephanus longispina (Schulz) Bukry \& Foster}

$$
\text { (Pl. 2, Fig. 8) }
$$

Distephanus crux f. longispina Schulz, 1928, p. 256, fig. 44; Ling, 1972, pl. 26, figs. 17-19.

Distephanus longispina (Schulz) Bukry and Foster, 1973, p. 844, pl. 4, figs. 7,8 .
Description: This four-sided Distephanus has a round or square basal ring with extra long spines along the major axis. It closely resembles $D$ s. crux, and is different only in that the major axis spines are greater than three-fourths the length of the basal ring, whereas the spine length of Ds. crux is less than onehalf the length of the basal ring.

\section{Distephanus speculum binoculus (Ehrenberg) Bukry}

(Pl. 3, Fig. 6)

Dictyocha binoculus Ehrenberg, 1844, pp. 63, 79.

Distephanus speculum binoculus (Ehrenberg) Bukry, 1975, p. 855; McCartney and Wise, 1987, p. 811, pl. 6, fig. 6.

Description: This taxon has a basal ring that is similar to that of $D s$. speculum speculum with six sides and six spines radiating from each vertex. The apical structure has two apical windows with six struts connecting the apical ring to the basal ring. Basal spikes on the basal ring are common.

\section{Distephanus speculum hemisphaericus (Ehrenberg) Bukry} (Pl. 3, Figs. 3, 4)

Dictyocha hemisphaerica Ehrenberg, 1844, pl. 17, fig. 5.

Distephanus speculum hemisphaericus (Ehrenberg) Bukry, 1975, p. 855, pl. 4 , fig. 8.

Description: This species includes specimens that have a six-sided basal ring with six radiating spines, two of the spines are longer along the major axis. The apical structure consists of three to seven windows that are connected to the basal ring by six struts. The designation Ds. speculum hemisphaericus include specimens of Ds. speculum haliomma as in Bukry, 1978a.

\section{Distephanus speculum patulus Bukry}

(Pl. 3, Fig. 7)

Distephanus speculum patulus (Ehrenberg) Bukry, 1982, p. 441, figs. 7-10.

Description: This taxon has a six-sided basal ring like that of Ds. speculum speculum, but has shorter spines, a larger apical ring, and no basal spikes.

\section{Distephanus speculum pentagonus Lemmermann}

(Pl. 3, Fig. 5)

Distephamus speculum var. pentagonus Lemmermann, 1901, p. 264, pl. 11, fig. 19; Bukry, 1976a, p. 895; McCartney and Wise, 1987, p. 811, pl. 8, fig. 8 .

Description: This five-sided Distephanus has five equant radiating spines and a five-sided apical ring that is connected to the basal ring by five struts, and the basal ring has no spikes. These specimens are common in the lower Miocene and uppermost Oligocene.

\section{Distephanus speculum speculum (Ehrenberg) Glezer}

$$
\text { (Pl. 2, Figs. 11, 12; Pl. 3, Figs. 1, 2) }
$$

Dictyocha speculum Ehrenberg, 1839, p. 150.

Distephanus speculum speculum (Ehrenberg) Glezer, 1966, p. 282, pl. 19, figs. 7-9.

Description: This taxon has a six-sided basal ring with six spines radiating from each corner of the basal ring. Two opposing spines are commonly longer than the other four spines, and the basal ring commonly has basal spikes. The apical ring has six sides and is attached to the basal ring by six struts that bisect each side of the basal ring.

\section{Distephanus stradneri (Jerkovic) Bukry}

(Pl. 2, Fig. 7)

Dictyocha schauinslandii stradneri Jerkovic, 1965, pp. 56, 76; Ehrenberg, 1854 , pl. 33, fig. 11

Distephanus stradneri (Jerkovic) Bukry, 1978a, p. 698. 
Description: Four-sided Distephanus with four spines, two longer spines along the major axis and two short spines along the minor axis. The apical window is less than one-half the size of the basal ring and is square. It is generally larger than Ds. crux and has basal spikes. This skeleton of this taxon is very symmetrical with a robust skeleton.

\section{Distephanus stradneri var. grandis Bukry}

(P1. 2, Figs. 9, 10)

Distephanus stradneri var. grandis Bukry, 1985, p. 495, pl. 4, figs. 1-7.

Description: Distephanus stradneri var. grandis is a four-sided silicoflagellate with four radial spines at each of the vertices and a small apical window. The vertices are angular and where the struts meet the basal ring it is indented. This taxon is similar to Ds. hannai, but differs in its larger size, and its angular vertices.

Genus NAVICULOPSIS Frenguelli, 1940

Naviculopsis biapiculata (Lemmermann) Frenguellii

?Dictyocha navicula biapiculata Lemmermann, 1901, p. 258, pl. 10, figs. 14, 15.

Naviculopsis biapiculata (Lemmermann) Frenguellii, 1940, p. 60, fig. 11c, d; Bukry, 1976a, pp. 896-897.

Description: This species has a small apical ring that is rounded where the basal ring and apical bar meet. The spines occur parallel to the major axis and vary in length, and the apical bar is narrow and greatly elevated compared to the apical bars of $N$. biapiculata variety 2 and $N$. constricta.

\section{Naviculopsis biapiculata variety 1}

(P1. 4, Fig. 13)

Description: This taxon is very small with long spines, usually more than half the length of the basal ring. The area where the apical bar and the basal ring meet is not constricted, but is straight, unlike $N$. biapiculata that is rounded where the apical bar and basal ring meet. This species looks very much like Naviculopsis constricta variety 2, but differs only by the fact than Naviculopsis constricta variety 2 has constriction where the basal ring meets the apical bar.

\section{Naviculopsis biapiculata variety 2}

(Pl. 4, Figs. 1, 2, 11)

Naviculsopsis eobiapiculata Bukry, 1978c, p. 799, pl. 4, figs. 9, 10, 15, 16 (not 11-14).

Naviculopsis biapiculata (Lemmermann) Frenguellii, Weaver and Spaw, 1978.

Naviculopsis biapiculata s.l. (Lemmermann) Frenguellii, Perch-Nielsen, 1975, p. 714, pl. 12, figs. 18, 20, 21 (not fig. 19).

Description: The designation $N$. biapiculata variety 2 includes a variety of Naviculopsis specimens that have an oval basal ring and can be either straight or bowed out where the apical bar meets the basal ring, as listed in the above references. It has two spines radiating along the major axis which vary in length from over one-half the major axis length to longer than the basal ring. It has a narrow apical bar with only slight, if any, constriction where the apical bar meets the basal ring.

\section{Naviculopsis biapiculata variety 3}

(Pl. 4, Figs. 4, 10)

Description: This designation includes all specimens that have a small, oblong basal ring and two spines along the major axis. The spines are almost the same length as the basal ring. Naviculopsis biapiculata variety 3 is similar to $N$. biapiculata variety 2 , but differs in that the apical bar is wider and the size of the specimen is generally smaller.

\section{Naviculopsis biapiculata nodulifera Ciesielski}

(Pl. 4, Figs. 8, 9)

Naviculopsis biapiculata nodulifera Ciesielski, 1991, p. 82, pl. 10, figs. 6, 7.
Description: This taxon has the same morphology as $N$. biapiculata and differs only by a small, almost unnoticeable nodule that protrudes from the center of the apical bar.

\section{Naviculopsis constricta (Schulz) Frenguellii}

(P1. 4, Fig. 3)

Dictyocha navicula biapiculata constricta Schulz, 1928, p. 246, fig. 21. Naviculopsis constricta (Schulz) Frenguellii, 1940, figs. 11a, b; Bukry, 1975, p. 872 , pl. 7 , figs. $1-3$.

Description: Naviculopsis species with a very long basal ring and very long spines, commonly equal to or longer than the length of the basal ring. The apical bar is commonly wider than, or, at times, the same width as the basal ring bars, and there is constriction where the apical bar meets the basal ring.

\section{Naviculopsis constricta variety 1}

(P1. 4, Fig. 7)

Description: Naviculopsis constricta variety 1 has a very long basal ring with two short spines along the major axis. The apical bar can be narrow or wide, but is commonly wide with constriction where the basal ring meets the apical bar.

\section{Naviculopsis constricta variety 2}

(P1. 4, Fig. 6)

Description: Specimens of Naviculopsis constricta variety 2 have a narrow and elongate basal ring with very pronounced constriction where the apical bar meets the basal ring. It commonly has a wider apical bar than $N$. biapiculata variety 2 , although the width of the apical bar may vary. The specimens have a shorter basal ring than $N$. constricta and also shorter spines.

\section{Naviculopsis contraria Bukry}

Naviculopsis contraria Bukry, 1982, p. 442, pl. 6, figs. 5-13; Bukry, 1985, p. 497, pl. 6 , fig. 1 .

Remarks: Only four specimens of this taxon were found in Sample 159959A-28X-4, 59-61 cm, in the $N$. ponticula Zone of the lower Miocene cores.

\section{Naviculopsis foliacea tumida Bukry}

$$
\text { (P1. 4, Fig. 5) }
$$

Naviculopsis foliacea tumida Bukry, 1978b, p. 841, pl. 8, figs. 1-8.

Naviculopsis lata (Deflandre) Frenguellii

$$
\text { (P1. 3, Fig. 11) }
$$

Dictyocha biapiculata var. lata Deflandre, 1932, Soc. Bot. France, Bull., v. 79, p. 500, figs. 30, 31.

Naviculopsis lata (Deflandre) Frenguelli, 1940, p. 61, fig. 11h; Ling, 1972, pl. 30, figs.12-14; Bukry, 1982, p. 443, pl. 7, figs. 11-14.

Description: This Naviculopsis has a round and stout basal ring with two short spines radiating along the long axis. Where the apical bar meets the basal ring the sides are straight or only slightly indented. This taxon has a larger basal ring than other Naviculopsis forms discussed.

\section{Naviculopsis lata variety 1}

Description: Specimens designated Naviculopsis lata variety 1 include all those with a large basal ring, a narrow apical bar, and basal spines that are from one-fourth to one-half the length of the major axis.

\section{Naviculopsis lata variety 2}

Description: This species differs from $N$. lata in that the area where the apical bar meets the basal ring is bowed out; whereas $N$. lata has sides that are straight or slightly indented. This species differs from $N$. biapiculata in that its spines are shorter and the basal ring is larger, the size of N. lata.

\section{Naviculopsis obtusarca Bukry}

(Pl. 3, Figs. 10, 12) 
Naviculopsis obtusarca Bukry, 1978b, p. 821.

$$
\text { Naviculopsis ponticula (Ehrenberg) Bukry }
$$

(Pl. 3, Fig. 9)

Dictyocha ponticulus Ehrenberg, 1844b, pp. 258, 267.

Naviculopsis ponticula (Ehrenberg) Bukry, 1978b, p. 821; McCartney and Wise, 1987, p. 814, pl. 5. figs. 9a, b.

Naviculopsis ponticula spinosa Bukry

(Pl. 3, Fig. 13)

Naviculopsis ponticula spinosa Bukry, 1982, p. 445, pl. 9, figs. 2-6.

\section{REFERENCES}

Bachmann, A., 1967. In Ichikawa, W., Shimizu, I., and Bachmann, A. (Eds.), Fossil Silicoflagellates and Their Associated Uncertain Forms in Iida Diatomite, Noto Peninsula, Central Japan. Sci. Rep. Kanazawa Univ., 12:143-172.

Bachmann, A., and Ichikawa, W., 1962. The silicoflagellides in the Wakura Beds, Nanao City, Prefecture Ishikawa, Japan. Sci. Rep. Kanazawa Univ., 3:161-175.

Bukry, D., 1973. Coccoliths and silicoflagellates from Deep Sea Drilling Project Leg 19, North Pacific Ocean and Bering Sea. In Creager, J.S., Scholl, D.W., et al., Init. Repts. DSDP, 19: Washington (U.S. Govt. Printing Office), 857-867.

-, 1974. Stratigraphic value of silicoflagellates in non-tropical regions. Bull. Geol. Soc. Am., 85:1905-1906.

, 1975. Silicoflagellate and coccolith stratigraphy, Deep Sea Drilling Project, Leg 29. In Kennett, J.P., Houtz, R.E., et al., Init. Repts. DSDP, 29: Washington (U.S. Govt. Printing Office), 845-872.

1976a. Cenozoic silicoflagellate and coccolith stratigraphy, South Atlantic Ocean, Deep Sea Drilling Project Leg 36. In Hollister, C.D., Craddock, C., et al., Init. Repts. DSDP, 35: Washington (U.S. Govt. Printing Office), 885-917.

1976b. Silicoflagellate and coccolith stratigraphy, southeastern Pacific Ocean, Deep Sea Drilling Project Leg 34. In Yeats, R.S., Hart, S.R., et al., Init. Repts. DSDP, 34: Washington (U.S. Govt. Printing Office), 715-735

— 1978a. Cenozoic coccolith and silicoflagellate stratigraphy, offshore northwest Africa, Deep Sea Drilling Project Leg 41. In Lancelot, Y., Seibold, E., et al., Init. Repts. DSDP, 41: Washington (U.S. Govt. Printing Office), 689-707.

1978b. Cenozoic coccolith, silicoflagellate, and diatom stratigraphy, Deep Sea Drilling Project Leg 44. In Bensen, W.E., Sheridan, R.E., et al., Init. Repts. DSDP, 44: Washington (U.S. Govt. Printing Office), 807-863.

_ $1978 \mathrm{c}$. Cenozoic silicoflagellate and coccolith stratigraphy, northwestern Atlantic Ocean, Deep Sea Drilling Project Leg 43. In Bensen, W.E., Sheridan, R.E., et al., Init. Repts. DSDP, 44: Washington (U.S. Govt. Printing Office), 775-805.

— 1978d. Cenozoic silicoflagellate and coccolith stratigraphy, southeastern Atlantic Ocean, Deep Sea Drilling Project Leg 40. In Bolli, H.M., Ryan, W.B.F., et al., Init. Repts. DSDP, 40: Washington (U.S. Govt. Printing Office), 635-649.

1979a. Coccolith and silicoflagellate stratigraphy, northern MidAtlantic Ridge and Reykjanes Ridge, Deep Sea Drilling Project Leg 49. In Luyendyk, B.P., Cann, J.R., et al., Init. Repts. DSDP, 49: Washington (U.S. Govt. Printing Office), 551-581.

1979b. Comments on opal phytoliths and stratigraphy of Neogene silicoflagellates and coccoliths at Deep Sea Drilling Project Site 397 off northwest Africa. In Luyendyk, B.P., Cann, J.R., et al., Init. Repts. DSDP, 49: Washington (U.S. Govt. Printing Office), 977-1009.

- 1980. Silicoflagellate biostratigraphy and paleoecology in the eastern equatorial Pacific, Deep Sea Drilling Project Leg 54. In Rosendahl, B.R., Hekinian, R., et al., Init. Repts. DSDP, 54: Washington (U.S. Govt. Printing Office), 545-573.

, 1981. Synthesis of silicoflagellate stratigraphy for Maestrichtian to Quaternary marine sediments. In Warme, T.E., Douglas, R.C., and Winterer, E.L. (Eds.), The Deep Sea Drilling Project: A Decade of Progress. Spec. Publ.—Soc. Econ. Paleontol. Mineral., 32:433-444.
, 1982. Cenozoic silicoflagellates from offshore Guatemala, Deep Sea Drilling Project Site 495. In Aubouin, J., von Huene, R., et al., Init. Repts. DSDP, 67: Washington (U.S. Govt. Printing Office), 425-445.

1985. Tropical Pacific silicoflagellate zonation and paleotemperature trends of the late Cenozoic. In Mayer, L., Theyer, F., Thomas, E., et al., Init. Repts. DSDP, 85: Washington (U.S. Govt. Printing Office), $477-$ 497.

, 1987. Eocene siliceous and calcareous phytoplankton, Deep Sea Drilling Project Leg 95. In Poag, C.W., Watts, A.B., et al., Init. Repts. DSDP, 95: Washington (U.S. Govt. Printing Office), 395-415.

Bukry, D., and Foster, J.H., 1973. Silicoflagellate and diatom stratigraphy, Leg 16, Deep Sea Drilling Project. In van Andel, T.H., Heath, G.R., et al., Init. Repts. DSDP, 16: Washington (U.S. Govt. Printing Office), 815871.

Ciesielski, P.F., 1991. Biostratigraphy of diverse silicoflagellate assemblages from the early Paleocene to early Miocene of Holes 698A, 700B, 702B, and 703A: subantarctic South Atlantic. In Ciesielski, P.F., Kristoffersen, Y., et al., Proc. ODP, Sci. Results, 114: College Station, TX (Ocean Drilling Program), 49-96.

Deflandre, G., 1950. Contribution a l'étude des silicoflagellidés actuels et fossiles. Microscopie, 2:72-108, 117-142, 191-210.

Ehrenberg, C.G., 1839. Über die Bildung der Kreidefelsen und des Kreidemergels durch sichtbare Organismen. Abh. Kgl. Preuss. (Akad. Wiss. Berlin), 59-147.

1840. 274 Blatter von ihm selbst ausgefuhrter Zeichnungen von ebenso vielen Arten. K. Preuss. Akad. Wiss. Berlin Ber., 1840:197-219.

, 1844. Über 2 neue Lager von Gebirgsmassen aus Infusorien als Meeres-Absatz in Nord-Amerika und eine Vergleichung derselben mit den organischen Kreide-Gebilden in Europa und Afrika. K. Preuss. Akad. Wiss. Berlin, Berichte, 57-97.

Frenguelli, J., 1940. Consideraciones sobre los silicoflagelados fósiles. Rev. Mus. La Plata, Secc. Geol., 2:37-112.

Glezer, Z.I., 1966. Silicoflagellatophyceae. In Gollerbakh, M.M. (Ed.), Cryptogamic Plants of the U.S.S.R. Akad. Nauk. SSSR, V.A. Komarova Bot. Inst. Translated 1970, Jerusalem (Israel Program Sci. Transl. Ltd.), 7:1363.)

Hanna, G.D., 1928. Silicoflagellates from the Cretaceous of California. $J$. Paleontol., 1:259-264

Hanna, G.D., 1931. Diatoms and silicoflagellates of the Kreyenhagen Shale. California Div. Mines State Mineral. Rept., 27:187-201.

Jerkovic, L., 1965. Sur Quelques Silicoflagellidés de Yugoslavie: Paris (Lab. Micropaleontol., Inst. Paleontol. Museum), 1-8.

Keller, G., and Barron, J.A., 1983. Paleoceanographic implications of Miocene deep-sea hiatuses. Geol. Soc. Am. Bull., 94:590-613.

Lemmermann, E., 1901. Silicoflagellatae. Ber. Dtsch. Bot. Ges., 19:247-271.

Ling, H.Y., 1972. Upper Cretaceous and Cenozoic silicoflagellates and ebridians. Bull Am. Paleontol., 62:135-229.

Locker, S., 1974. Revision der Silicoflagellaten aus der Mikrogeologischen Sammlung von C.G. Ehrenberg. Eclogae Geol. Helv., 67:631-646.

, 1975. Dictyocha varia sp. n., eine miozäne Silicoflagellaten-Art mit kompliziertem Variationsmodus. Akad. Geol. Wiss., 3:99-103.

Locker, S., and Martini, E., 1986. Silicoflagellates and some sponge spicules from the southwest Pacific, DSDP Leg 90. In Kennett, J.P., von der Borch, C.C., et al., Init. Repts. DSDP, 90: Washington (U.S. Govt. Printing Office), 887-924.

Loeblich, A., III, Loeblich, L.A., Tappan, H., and Loeblich, A.R., Jr., 1968. Annotated index of fossil and recent silicoflagellates and ebridians with descriptions and illustrations of validly proposed taxa. Mem.-Geol. Soc. Am., 106:1-319.

Martini, E., 1971. Neogene silicoflagellates from the equatorial Pacific. In Winterer, E.L., Riedel, W.R., et al., Init. Repts. DSDP, 7 (Pt. 2): Washington (U.S. Govt. Printing Office), 1695-1708.

- 1972. Silicoflagellate zones in the late Oligocene and early Miocene of Europe. Senckenbergiana Lethaea, 53:119-122.

Martini, E., and Müller, C., 1976. Eocene to Pleistocene silicoflagellates from the Norwegian-Greenland Sea (DSDP Leg 38). In Talwani, M., Udintsev, G., et al., Init. Repts. DSDP, 38: Washington (U.S. Govt. Printing Office), 857-895.

Mascle, J., Lohmann, G.P., Clift, P.D., et al., 1996. Proc. ODP, Init. Repts., 159: College Station, TX (Ocean Drilling Program).

McCartney, K., Churchill, S., and Woestendiek, L., 1995. Silicoflagellates and ebridians from Leg 138, Eastern Equatorial Pacific. In Pisias, N.G., Mayer, L.A., Janecek, T.R., Palmer-Julson, A., and van Andel, T.H. 
(Eds.), Proc. ODP, Sci. Results, 138: College Station, TX (Ocean Drilling Program), 129-162.

McCartney, K., and Wise, S.W., Jr., 1987. Silicoflagellates and ebridians from the New Jersey Transect, Deep Sea Drilling Project Leg 93, Sites 604 and 605. In van Hinte, J.E., Wise, S.W., Jr., et al., Init. Repts. DSDP, 93 (Pt. 2): Washington (U.S. Govt. Printing Office), 801-814.

Mikkelsen, N., and Barron, J.A., 1997. Early Oligocene Diatoms on the Ceara Rise and the Cenozoic evolution of biogenic silica accumulation in the low latitude Atlantic. In Shackleton, N.J., Curry, W.B., Richter, C., and Bralower, T.J. (Eds.), Proc. ODP, Sci. Results, 154: College Station, TX (Ocean Drilling Program), 483-490.

Perch-Nielsen, K., 1975. Late Cretaceous to Pleistocene silicoflagellates from the southern Southwest Pacific, DSDP, Leg 29. In Kennett, J.P., Houtz, R.E., et al., Init. Repts. DSDP, 29: Washington (U.S. Govt. Printing Office), 677-721.

, 1985. Silicoflagellates. In Bolli, H.M., Saunders, J.B., and PerchNielsen, K. (Eds.), Plankton Stratigraphy: Cambridge (Cambridge Univ. Press), 811-846.

Schulz, P., 1928. Beitrage zur Kenntinis fossiler und rezenter silicoflagellaten. Bot. Archiv., 21:225-292.
Shaw, C.A., and Ciesielski, P.F., 1983. Silicoflagellate biostratigraphy of middle Eocene to Holocene subantarctic sediments recovered by Deep Sea Drilling Project Leg 71. In Ludwig, W.J., Krasheninnikov, V.A., et al., Init. Repts. DSDP, 71 (Pt. 2): Washington (U.S. Govt. Printing Office), 687-737.

Stein, R., 1985. The post-Eocene sediment record of DSDP Site 316: implications for African climate and plate tectonic drift. Mem.-Geol. Soc. Am., 163:305-315.

Weaver, F.M., and Spaw, J.M., 1978. Current Concepts in Silicoflagellate Biostratigraphy. Exxon Prod. Res., EPR.8EX.78.

Woodruff, F., and Savin, S.M., 1989. Miocene deepwater oceanography, Paleoceanography, 4:87-140.

Date of initial receipt: 16 September 1996

Date of acceptance: 4 April 1997

Ms 159SR-025 

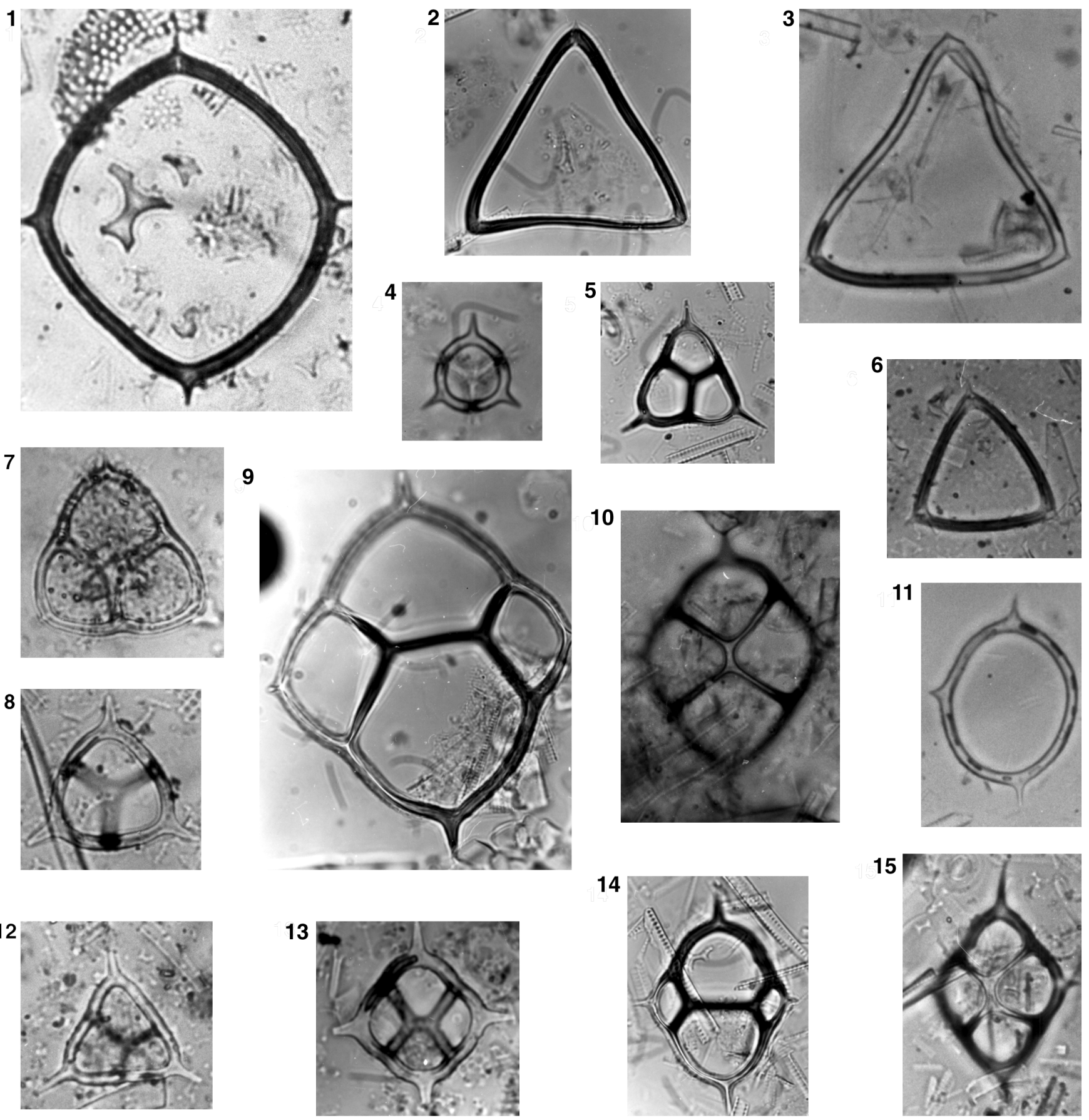

11
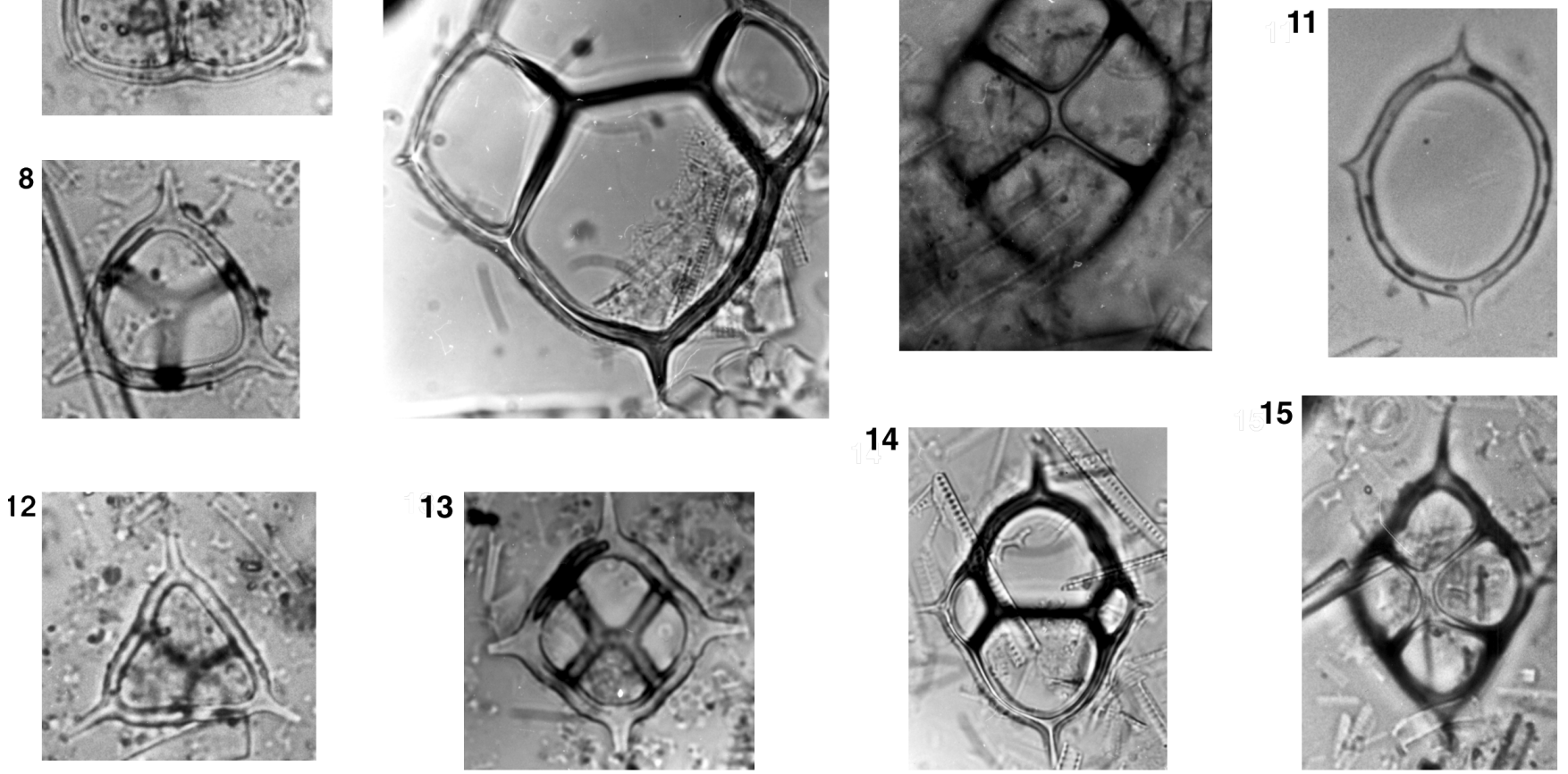

\section{$20 \mu \mathrm{m}$}

Plate 1. Silicoflagellates from Leg 159. 1. Bachmannocena elliptica (Ehrenberg) Ehrenberg, Sample 159-959A-27X-5, 23-25 cm. 2, 6. Bachmannocena apiculata apiculata (Schulz) Bukry, Samples 159-959A-35X-3, 59-61 cm, and 159-959A-33X-CC. 3. Bachmannocena apiculata glabra (Schulz) Bukry, Sample 159-959A-33X-4, 58-59 cm. 4. Dictyocha spinosa (Deflandre) Glezer, Sample 159-959A-28X-CC. 5. Corbisema triacantha triacantha (Ehrenberg) Bukry, Sample 159-959A-38X-5, 71-73 cm. 7. Corbisema apiculata (Lemmermann) Hanna, Sample 159-959D-1R-CC. 8. Corbisema triacantha variety 1, Sample 159-959A-44X-5, 35-37 cm. 9. Dictyocha fibula fibula Ehrenberg, Sample 159-959A-30X-7, 36-38 cm. 10, 15. Dictyocha extensa extensa (Locker) Locker and Martini, Sample 159-959A-23X-6, 89-91 cm. 11. Bachmannocena triodon Bukry, Sample 159-959A-23X-6, 89-91 cm. 12. Corbisema triacantha mediana Bukry, Sample 159-959D-1R-CC. 13. Dictyocha deflandrei Frenguellii (1940) ex Glezer, Sample 159-959A-45X-3, 101-102 cm. 14. Dictyocha fibula ausonia (Deflandre) McCartney et al., Sample 159-959A-24X-2, 59-60 cm. 

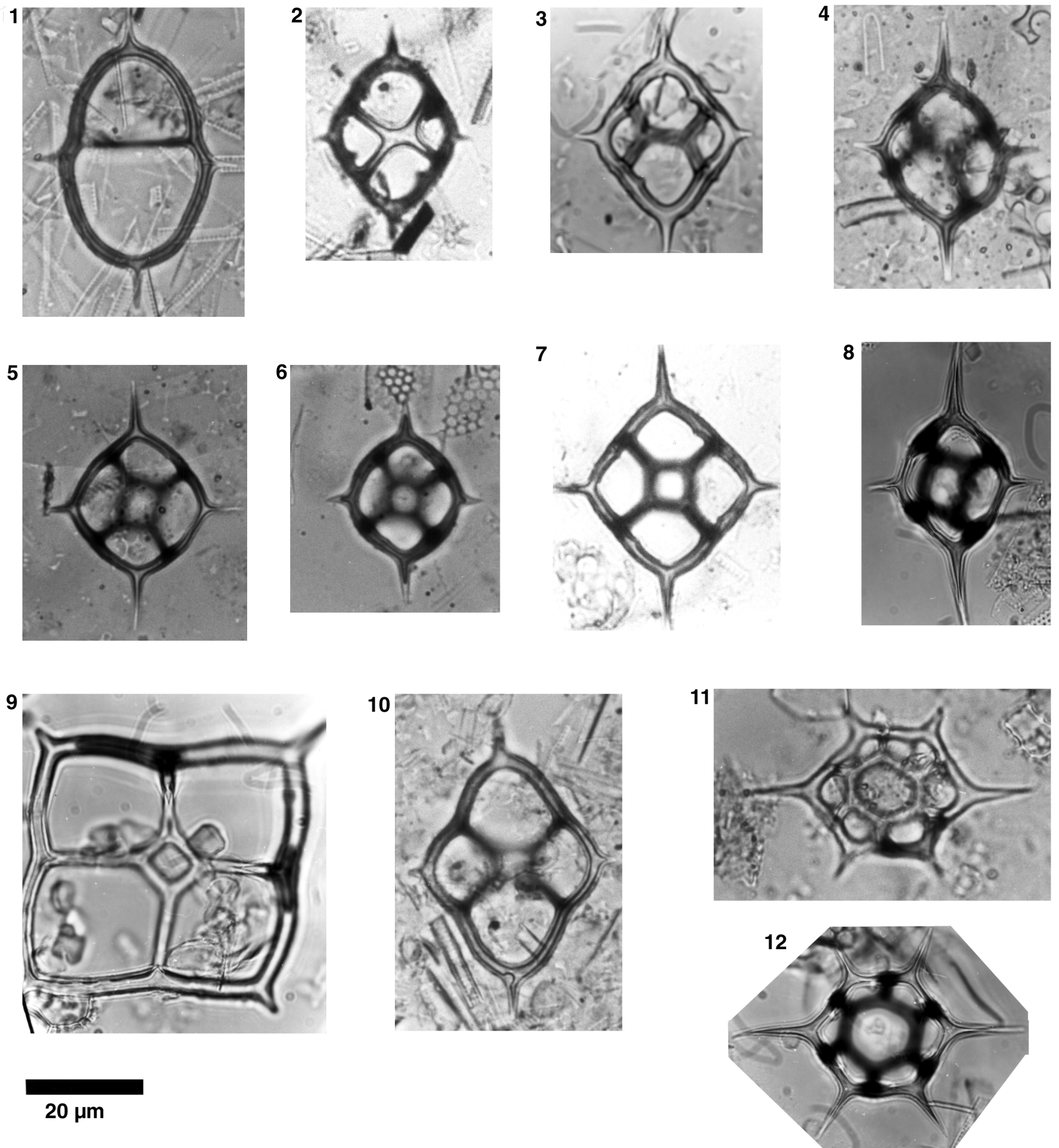

Plate 2. Silicoflagellates from Leg 159. 1. Dictyocha fibula ausonia (Deflandre) (naviculopsoid) McCartney et al., Sample 159-959A-24X-2, 59-60 cm. 2, 3. Dictyocha varia Locker, Sample 159-959A-23X-6, 89-91 cm. 4. Dictyocha species 1, Sample 159-959A-31X-CC. 5, 6. Distephanus crux Ehrenberg, Samples 159-959A-28X-3, 9-11 cm, and 159-959A-36X-3, 88-89 cm. 7. Distephanus stradneri Jerkovic, Sample 159-959A-27X-5, 23-25 cm. 8. Distephanus longispinus (Schulz) Bukry and Foster, Sample 159-959A-34X-CC. 9, 10. Distephanus stradneri var. grandis Bukry, Sample 159-959A-34X-CC. 11, 12. Distephanus speculum speculum (Ehrenberg) Glezer, Samples 159-959A-36X-3, 88-89 cm, and 159-959A-33X-4, 58-59 cm. 

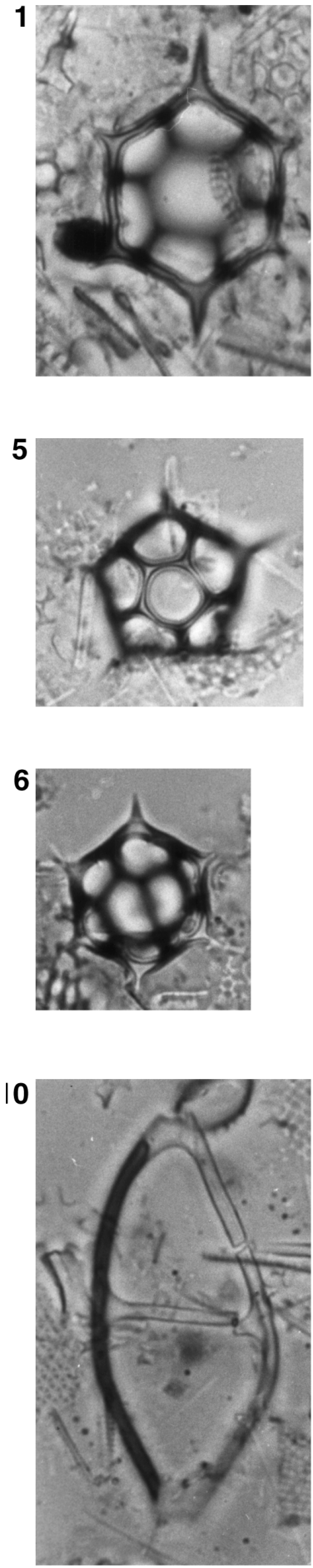
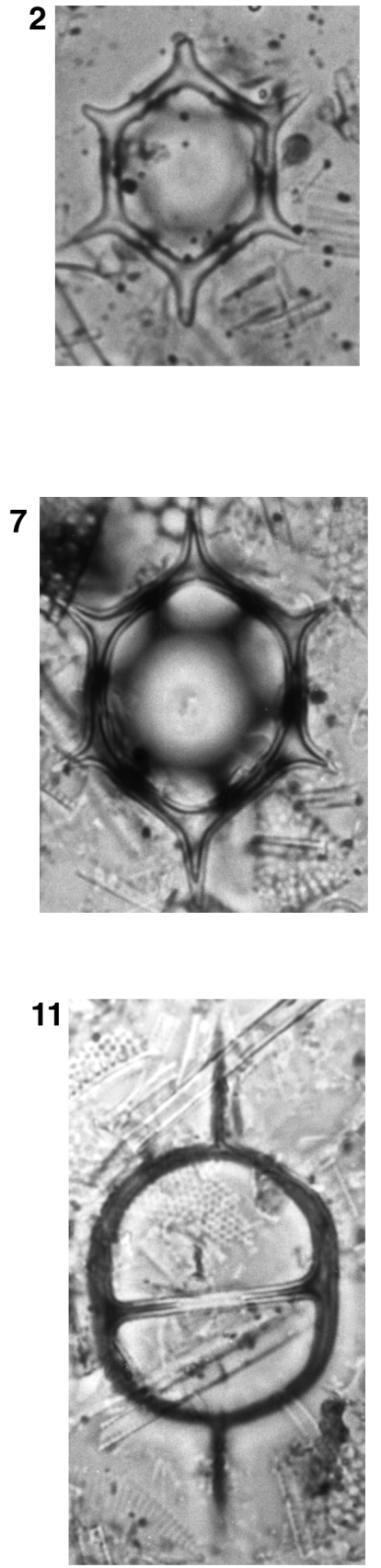

$20 \mu \mathrm{m}$
3

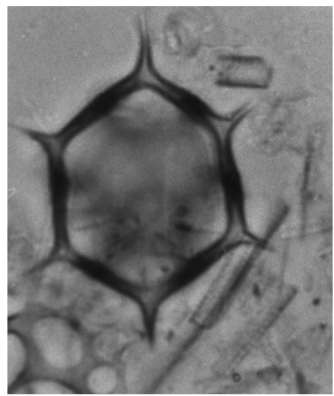

8

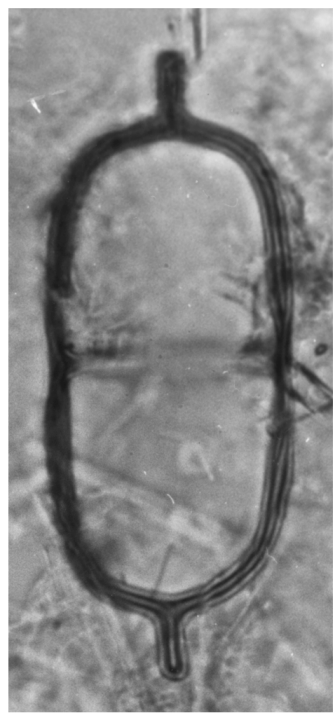

12

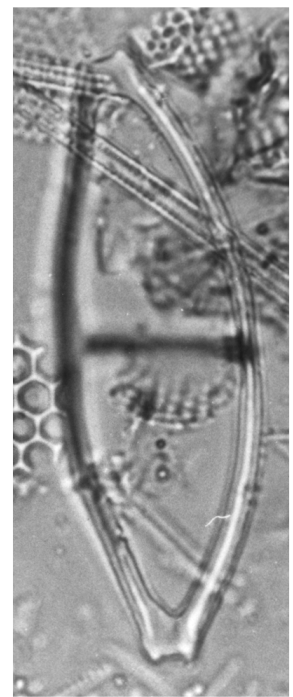

4

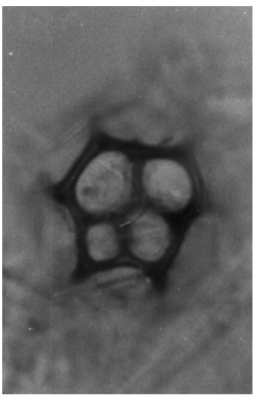

9

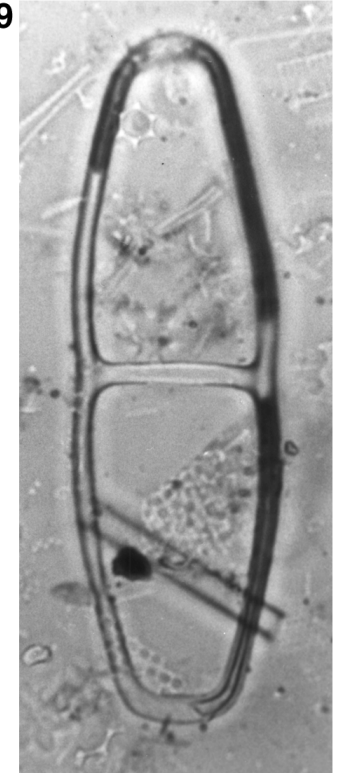

13

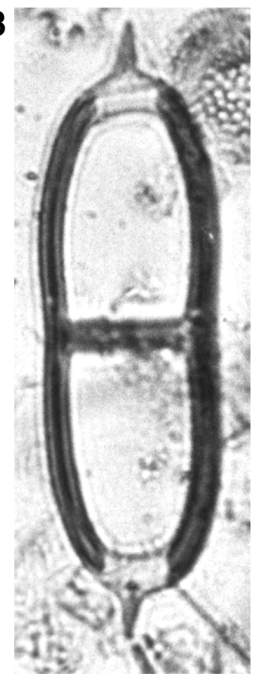

Plate 3. Silicoflagellates from Leg 159. 1, 2. Distephanus speculum speculum (Ehrenberg) Glezer, Samples 159-959A-34X-CC and 159-959A-32X-1, 49-51 cm. 3, 4. Distephanus speculum hemisphaericus (Ehrenberg) Bukry, Sample 159-959A-23X-6, 89-91 cm. 5. Distephanus speculum pentagonus Lemmermann, Sample 159-959A-27X-3, 37-38 cm. 6. Distephanus speculum binoculus (Ehrenberg) Bukry, Sample 159-959A-27X-3, 37-38 cm. 7. Distephanus speculum patulus Bukry, Sample 159-959A-27X-3, 37-38 cm. 8. Naviculopsis quadrata (Ehrenberg) Locker, Sample 159-959A-34X-CC. 9. Naviculopsis ponticula (Ehrenberg) Bukry, Sample 159-959A-28X-6, 106-108 cm. 10, 12. Naviculopsis obtusarca Bukry, Sample 159-959A-28X-6, 106-108 cm. 11. Naviculopsis lata Deflandre, Sample 159-959A-30X-7, 36-38 cm. 13. Naviculopsis ponticula spinosa Bukry, Sample 159-959A-27X-5, 23-25 cm. 

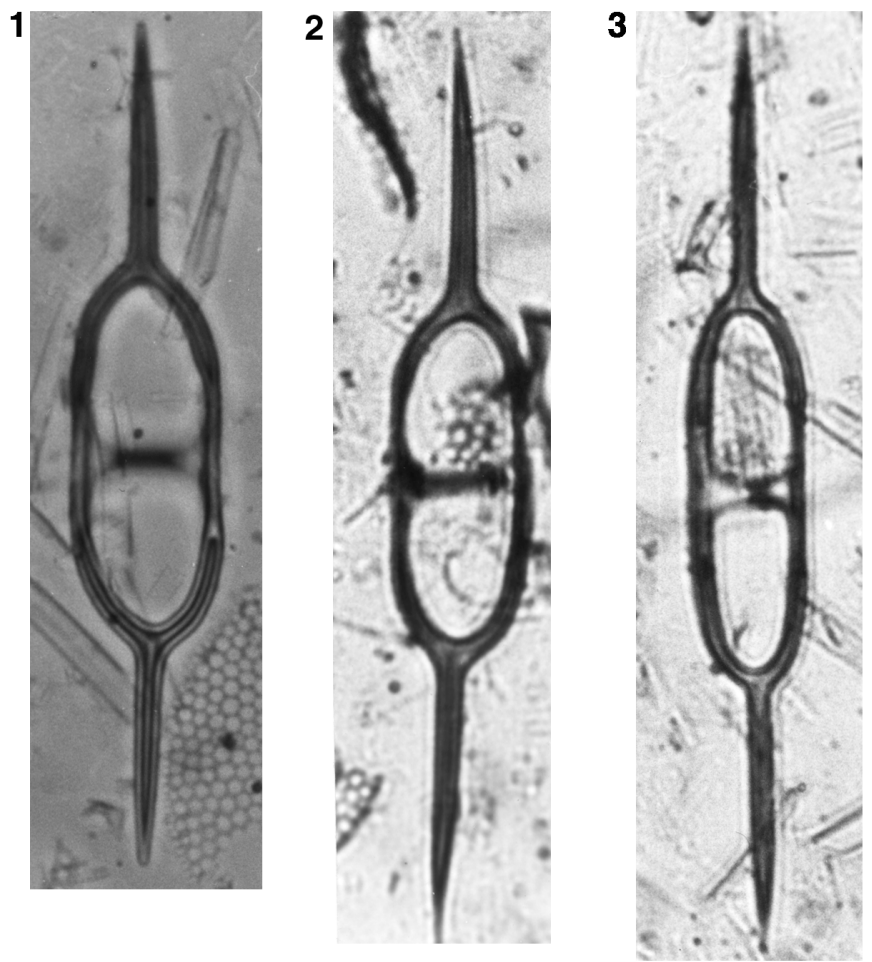

4

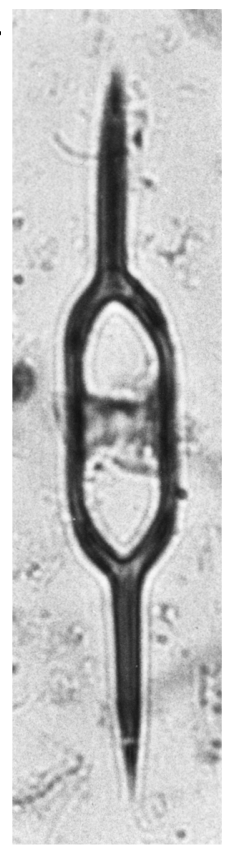

5

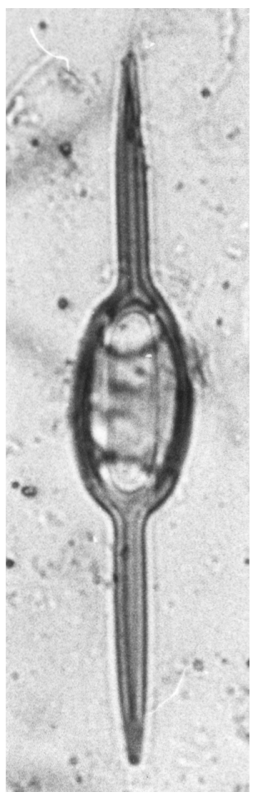

10

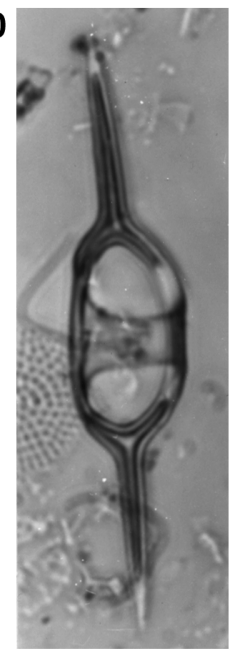

6

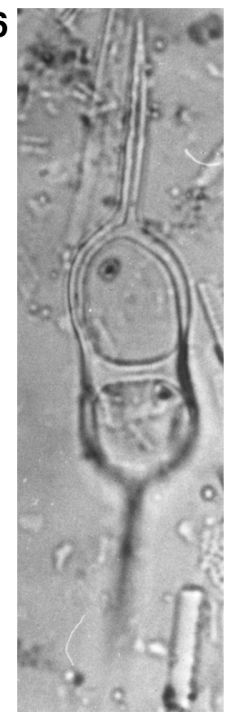

7

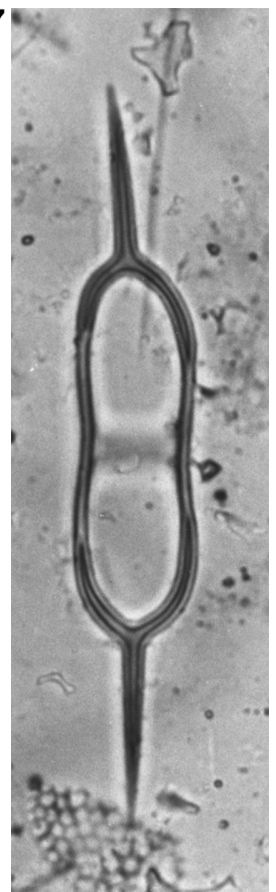

8

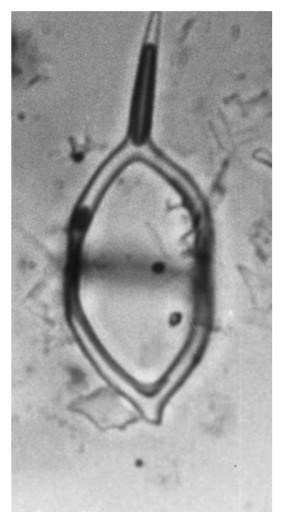

9

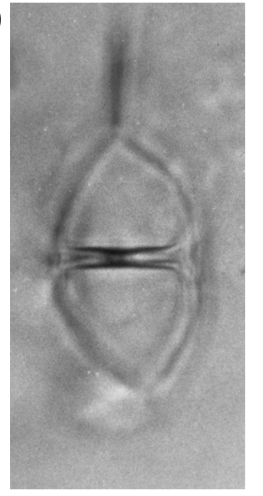

12

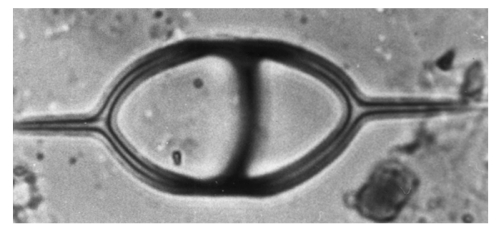

\section{1}

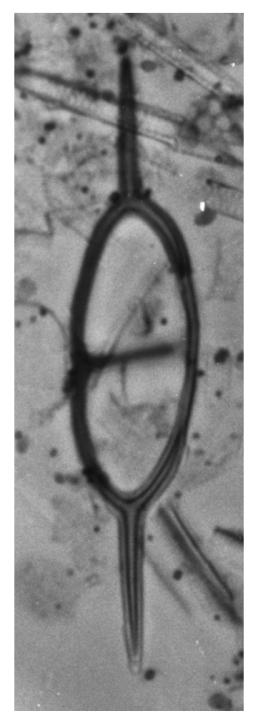

13

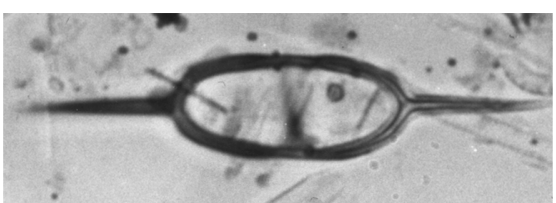

Plate 4. Silicoflagellates from Leg 159. 1, 2, 11. Naviculopsis biapiculata variety 2, Samples 159-959A-33X-6, 62-64 cm, 159-959A-38X-5, 71-73 cm, and 159-959A-33X-4, 58-59 cm. 3. Naviculopsis constricta (Schulz) Frenguellii, Sample 159-959A-33X-2, 149-150 cm. 4, 10. Naviculopsis biapiculata variety 3, Sample 159-959A-44X-1, 44-45 cm, and 159-959D-5R-CC, 1-2 cm. 5. Naviculopsis foliacea tumida Bukry, Sample 159-959D-7R-CC. 6. Naviculopsis constricta variety 2, Sample 159-959A-31X-7, 2-3 cm. 7. Naviculopsis constricta variety 1, Sample 159-959A-36X-3, 88-89 cm. 8, 9. Naviculopsis biapiculata nodulifera Ciesielski, Sample 159-959A-43X-2, 91-92 cm. 12. Naviculopsis biapiculata (Lemmermann) Frenguellii, Sample 159-959A-31X-2, 128-130 cm. 13. Naviculopsis biapiculata var. 1 , Sample $159-959 \mathrm{~A}-31 \mathrm{X}-7,2-3 \mathrm{~cm}$. 